TRANSACTIONS OF THE

AMERICAN MATHEMATICAL SOCIETY

Volume 362, Number 7, July 2010, Pages 3499-3533

S 0002-9947(10)04988-3

Article electronically published on February 15, 2010

\title{
SOME MODEL THEORY OF POLISH STRUCTURES
}

\author{
KRZYSZTOF KRUPIŃSKI
}

\begin{abstract}
We introduce a notion of Polish structure and, in doing so, provide a setting which allows the application of ideas and techniques from model theory, descriptive set theory, topology and the theory of profinite groups. We define a topological notion of independence in Polish structures and prove that it has some nice properties. Using this notion, we prove counterparts of some basic results from geometric stability theory in the context of small Polish structures. Then, we prove some structural theorems about compact groups regarded as Polish structures: each small, $n m$-stable compact $G$-group is solvable-by-finite; each small compact $G$-group of finite $\mathcal{N} M$-rank is nilpotentby-finite. Examples of small Polish structures and groups are also given.
\end{abstract}

\section{INTRODUCTION}

We propose a new, model theoretic approach to study classical descriptive set theoretic objects, like Polish $G$-spaces or Borel $G$-spaces. More generally, we are going to study Polish structures which are defined as follows.

Definition 1.1. A Polish structure is a pair $(X, G)$, where $G$ is a Polish group acting faithfully on a set $X$ so that the stabilizers of all singletons are closed subgroups of $G$. We say that $(X, G)$ is small if for every $n \in \omega$, there are only countably many orbits on $X^{n}$ under the action of $G$.

Notice that the assumption that $G$ acts faithfully on $X$ is purely cosmetic, as we can always divide $G$ by the maximal subgroup acting trivially on $X$.

Particular cases of Polish structures are profinite structures ( $X$ and $G$ are profinite and the action is continuous) introduced by Newelski in [21, 22, and then considered also by Wagner [15, 27] and by myself [10, 11, 12, 8, and, more generally, compact structures 13 ( $X$ is a compact metric space, $G$ is a compact group and the action is continuous). More generally, Polish $G$-spaces ( $X$ is Polish and the action is continuous) and Borel $G$-spaces ( $X$ is a standard Borel space and the action is Borel-measurable) are also examples of Polish structures. A typical

Received by the editors February 11, 2008.

2010 Mathematics Subject Classification. Primary 03C45, 03E15; Secondary 54H11, 20E18, $54 \mathrm{~F} 15$.

Key words and phrases. Polish structure, Polish group, profinite group, independence relation.

This research was supported by the Polish Government grant N201 $03232 / 2231$ and by NSF grant DMS 0300639.

(C)2010 American Mathematical Society Reverts to public domain 28 years from publication 
topological example of a Polish structure is any compact metric space $X$ together with the group $\mathrm{Homeo}(X)$ of all homeomorphisms equipped with the compact-open topology.

The general goal of this paper, as in [13] for the case of compact structures, is to make an attempt to apply stability-theoretic ideas to various classical mathematical objects. A similar motivation appears in [21, 22, where Newelski was considering small profinite structures. However, Polish structures (particularly Polish $G$-spaces) seem to be more interesting than profinite structures from the point of view of descriptive set theory and topology. Even when we look at profinite groups, it seems more natural to consider them together with the full group of topological automorphisms rather than with the group of automorphisms preserving a distinguished inverse system. Moreover, in my opinion, Polish structures yield a more adequate generalization of profinite structures than compact structures. That is because each small compact structure is profinite, so instead of smallness one should assume here the existence of $m$-independent extensions. But it is not easy to check whether this property holds in concrete examples, and it is difficult to find interesting examples of such compact structures which are not profinite. In contrast, we have many natural examples of small Polish structures which are not profinite.

In Section 2, we introduce the notion of $n m$-independence and we prove that it has some nice properties, similar to those of forking independence in stable theories. We show that $n m$-independence generalizes the notion of $m$-independence introduced by Newelski for profinite structures. However, the proof that $\mathrm{nm}$ independence is transitive is rather complicated, and it uses some descriptive set theory, whereas transitivity of $m$-independence follows immediately from the definition. Similar to the case of profinite structures, in order to get the existence of $n m$-independent extensions, we need to assume smallness. In fact, in many general results we could just assume the existence of $n m$-independent extensions (as in [13]), but we prefer to assume smallness since it is more natural and easier to check in concrete examples.

In Section 3, we consider counterparts of some basic notions from geometric stability theory and prove fundamental results about them. In particular, using $n m$-independence, we define $\mathcal{N} M$-rank in the same way as Lascar U-rank is defined by means of forking independence. Then, $\mathcal{N} M$-rank, satisfying Lascar inequalities, becomes a new tool that can be used to measure a topological complication of orbits.

In Section [4 we give examples of small Polish structures which are not profinite. In particular, we prove that the pseudo-arc considered with the full group of homeomorphisms is a small Polish structure.

In Section 5 , we study small topological groups regarded as Polish structures. We prove the existence of generics in a large class of such groups. Then, we study the structure of small compact $G$-groups (Definition 5.1). We give examples showing that counterparts of some conjectures about small profinite groups are false in our wider context. Then, we prove the main structural results: each small, $n m$-stable compact $G$-group is solvable-by-finite, and each small compact $G$-group of finite $\mathcal{N} M$-rank is nilpotent-by-finite. We finish with a list of open questions.

Having established all the basic notions and results, the next natural step would be to try to prove the counterparts of some deep results from stability theory, e.g. a group configuration theorem. Such results were proved by Newelski for small 
profinite structures [22]. In [13], the author noticed that most of them can be generalized to the case of compact [profinite] structures satisfying the existence of $m$-independent extensions. For small Polish structures, the situation is more complicated, and it is sometimes not clear how to formulate the appropriate conjectures.

There are also certain open questions about the existence of small profinite structures with some model theoretic properties (e.g. $\mathcal{M}$-gap conjecture [21, 22, 27]). It would be interesting to find counterexamples to their generalizations to the wider context of small Polish structures.

In Section 4 we give several examples of compact metric spaces with the full group of homeomorphisms which are small. A very general task could be to understand which compact metric spaces [continua] with the full group of homeomorphisms are small.

In Section 5, there are several specific questions about the structure of small Polish $G$-groups.

The notions introduced in this paper (e.g. $n m$-independence, $\mathcal{N} M$-rank) lead to new (motivated by model theory) questions and results concerning descriptive set theoretic and topological objects. On the other hand, they may also turn out to be new tools to deal with purely descriptive set theoretic or topological problems.

The author is very grateful to Sławomir Solecki for interesting discussions and suggestions, in particular, for bringing to his attention the pseudo-arc, which turned out to be an important example of a small Polish structure.

\section{INDEPENDENCE RELATION}

In this section, we define a notion of independence, which we call $\mathrm{nm}$-independence (read non-meager independence), and we study its properties. We also prove that it coincides with Newelski's $m$-independence in compact structures.

If $(X, G)$ is a Polish structure and $A \subseteq X$, then by $G_{A}$ we denote the pointwise stabilizer of $A$. For $a \in X^{n}$, we define $o(a / A)=\left\{f(a): f \in G_{A}\right\}$ (the orbit of $a$ over $A$ ).

Let us recall the definition of $m$-independence.

Definition 2.1. Let $(X, G)$ be a compact structure, $a$ be a finite tuple and $A, B$ be finite subsets of $X$. We say that $a$ is $m$-independent from $B$ over $A$ (written $\left.a^{m} \mathcal{L}_{A} B\right)$ if $o(a / A B)$ is open in $o(a / A)$. We say that $a$ is $m$-dependent on $B$ over $A$ (written $a^{m} \mathcal{L}_{A} B$ ) if $o(a / A B)$ is nowhere dense in $o(a / A)$.

We cannot use the above definition for a Polish structure $(X, G)$, as there is no topology on $X$. Even if we assumed that $(X, G)$ is a Polish $G$-space, orbits could be weird, e.g. meager in their relative topologies, and then $m$-independence would not have nice properties in this context.

The idea to avoid the above obstacle is to define a relation of independence in terms of the Polish group $G$.

Definition 2.2. Let $(X, G)$ be a Polish structure, $a$ be a finite tuple and $A, B$ be finite subsets of $X$. Let $\pi_{A}: G_{A} \rightarrow o(a / A)$ be defined by $\pi_{A}(g)=g a$. We say that $a$ is $n m$-independent from $B$ over $A$ (written $a \downarrow_{A}^{n m} B$ ) if $\pi_{A}^{-1}[o(a / A B)]$ is non-meager in $\pi_{A}^{-1}[o(a / A)]$. Otherwise, we say that $a$ is $n m$-dependent on $B$ over $A$ (written $\left.a \mathcal{X}_{A}^{n m} B\right)$.

One can also define $o$-independence by just replacing the word 'non-meager' by 'open' in the above definition. Some of the results will work for both notions of 
independence. However, the proof of the existence of $\mathrm{nm}$-independent extensions in small Polish structures does not work for $o$-independence. In Section 4, we will show that the pseudo-arc is an example of a small Polish structure without the existence of $o$-independent extensions. That is why $\mathrm{nm}$-independence is a more appropriate notion of independence.

Notice that $n m$ and $o$ in Definition 2.2 come from topological properties 'nonmeager' and 'open', whereas $m$ in Definition 2.1 comes from the word 'multiplicity'.

Notation. If $T$ is a topological space and $U, V \subseteq T$, then $U \subseteq_{n m} V$ means that $U$ is a non-meager subset of $V$ and $U \subseteq_{o} V$ means that $U$ is an open subset of $V$. When we write $*=n m$, it means that $*$ stands for non-meager; similarly, $*=o$ means that $*$ stands for open. Later we will also use this notation for $* \in\{c, m, n w d\}$, where $c$ stands for closed, $m$ for meager and $n w d$ for nowhere dense. Similarly, if $G$ is a topological group, then $H<_{*} G$ means that $H$ is a $*$-subgroup (not necessarily proper) of $G$, where $* \in\{o, n m, m, c, n w d\}$.

Proposition 2.3. Let $(X, G)$ be a Polish structure, a be a finite tuple and $A, B$ be finite subsets of $X$. Assume $*=n m$ or $*=o$. Then, TFAE:

(1) $a \mathbb{\Perp}_{A}^{*} B$,

(2) $G_{A B} G_{A a} \subseteq_{*} G_{A}$,

(3) $G_{A B} / G_{A a} \subseteq_{*} G_{A} / G_{A a}$

Proof. (1) $\Leftrightarrow(2)$. An easy computation shows that $\pi_{A}^{-1}[o(a / A C)]=G_{A C} G_{A a}$ for any $C \subseteq X$. Hence, $\pi_{A}^{-1}[o(a / A B)]=G_{A B} G_{A a}$ and $\pi_{A}^{-1}[o(a / A)]=G_{A}$. Now, the desired equivalence follows directly from the definition of $*$-independence.

$(2) \Rightarrow(3)$. Let $\pi: G_{A} \rightarrow G_{A} / G_{A a}$ be the quotient map. Then, $\pi$ is continuous and open.

If $*=o$, the implication follows from the fact that $\pi$ is open. So, consider the case $*=n m$. Suppose $G_{A B} / G_{A a}$ is a meager subset of $G_{A} / G_{A a}$, i.e. $G_{A B} / G_{A a}$ is covered by a countable union $\bigcup_{i \in \omega} D_{i}$ of closed and nowhere dense subsets of $G_{A} / G_{A a}$. Then, $G_{A B} G_{A a}=\pi^{-1}\left[G_{A B} / G_{A a}\right] \subseteq \bigcup_{i \in \omega} \pi^{-1}\left[D_{i}\right]$. Since $\pi$ is continuous and open, we get that $\pi^{-1}\left[D_{i}\right], i \in \omega$, are closed and nowhere dense. So, $G_{A B} G_{A a}$ is a meager subset of $G_{A}$.

$(3) \Rightarrow(2)$. If $*=o$, it follows from continuity of $\pi$. So, consider the case $*=n m$. Since $\pi$ is continuous and $G_{A B}$ is Polish, we see that $G_{A B} / G_{A a}$ is analytic so it has the Baire property [7, Theorem 21.6]; i.e. $G_{A B} / G_{A a}=D \triangle U$, where $D$ is meager and $U$ is open in $G_{A} / G_{A a}$. Assume now that $G_{A B} / G_{A a} \subseteq_{n m} G_{A} / G_{A a}$. Then, $U$ is non-empty. We have $G_{A B} G_{A a}=\pi^{-1}[D] \triangle \pi^{-1}[U]$. Since $\pi$ is continuous and open, we get that $\pi^{-1}[D]$ is meager and $\pi^{-1}[U] \neq \emptyset$ is open in $G_{A}$. Hence, $G_{A B} G_{A a} \subseteq_{n m} G_{A}$.

Definition 2.4. Let $(X, G)$ be a Polish structure and $A$ be a finite subset of $X$. We define the algebraic closure of $A$ (written $A c l(A)$ ) as the set of all elements of $X$ with countable orbits over $A$. The strong algebraic closure of $A$ (denoted by $\operatorname{acl}(A)$ ) is the set of all elements of $X$ with finite orbits over $A$. The definable closure of $A$ (written $d \operatorname{cl}(A))$ is the set of all elements of $X$ which are fixed by $G_{A}$. If $A$ is infinite, we define $\operatorname{Acl}(A)=\bigcup\left\{\operatorname{Acl}\left(A_{0}\right): A_{0} \subseteq A\right.$ is finite $\}, \operatorname{acl}(A)=\bigcup\left\{\operatorname{acl}\left(A_{0}\right)\right.$ : $A_{0} \subseteq A$ is finite $\}$ and $\operatorname{dcl}(A)=\bigcup\left\{d c l\left(A_{0}\right): A_{0} \subseteq A\right.$ is finite $\}$.

In Polish structures, Acl plays a similar role to acl in compact [profinite] structures. 
Theorem 2.5. In any Polish structure $(X, G), *$-independence, where $*=n m$ or $*=o$, has the following properties:

(0) (Invariance) $a \downarrow^{*}{ }_{A} B \Longleftrightarrow g(a) \downarrow_{g[A]}^{*} g[B]$ whenever $g \in G$ and $a, A, B \subseteq X$ are finite.

(1) (Symmetry) $a{\downarrow_{C}^{*}}_{C} b$ iff $b{L_{C}^{*}}_{C}$ a for every finite $a, b, C \subseteq X$.

(2) (Transitivity) $a \perp_{B}^{*} C$ and $a \downarrow^{*} B$ iff $a \perp^{*} C$ for every finite $A \subseteq B \subseteq C \subseteq$ $X$ and $a \subseteq X$.

(3) For every finite $A \subseteq X, a \in \operatorname{Acl}(A)$ iff for all finite $B \subseteq X$ we have $a \downarrow^{*}{ }_{A} B$.

Only (2) requires a serious argument, but let us first check (0), (1) and (3) for completeness.

Proof of (0), (1) and (3) in Theorem 2.5. (0) We know that $a \downarrow^{*}{ }_{A} B$ if and only if $G_{A B} G_{A a} \subseteq_{*} G_{A}$, which in turn is equivalent to $g G_{A B} g^{-1} g G_{A a} g^{-1} \subseteq_{*} g G_{A} g^{-1}$, and hence to $G_{g[A] g[B]} G_{g[A] g(a)} \subseteq_{*} G_{g[A]}$. The last condition is equivalent to $g(a) \perp_{g[A]}^{*} g[B]$.

(1) $a \perp^{*}{ }_{A} b \Longleftrightarrow G_{A b} G_{A a} \subseteq_{*} G_{A} \Longleftrightarrow G_{A a} G_{A b} \subseteq_{*} G_{A} \Longleftrightarrow b \perp_{A}^{*} a$.

(3) $(\Rightarrow)$. $a \in \operatorname{Acl}(A)$ means that $o(a / A)$ is countable. Hence, $\left[G_{A}: G_{A a}\right] \leq \omega$. So, $G_{A a}$ is non-meager in $G_{A}$ by the Baire category theorem. Since $G_{A a}$ is a closed subgroup of $G_{A}$, we get $G_{A a} \subseteq_{o} G_{A}$, and so $G_{A B} G_{A a} \subseteq_{o} G_{A}$ for any finite $B \subseteq X$. This implies that $a \perp^{*} B$ for all finite $B \subseteq X$.

$(\Leftarrow)$. Suppose that $a\rfloor_{A}^{*} B$ for every finite $B \subseteq X$. Then, $a \downarrow^{*} A a$. Hence, $G_{A a} \subseteq_{*} G_{A} \Longrightarrow G_{A a} \subseteq_{o} G_{A} \Longrightarrow\left[G_{A}: G_{A a}\right] \leq \omega \Longrightarrow a \in \operatorname{Acl}(A)$.

In order to prove transitivity, we first need to prove some purely descriptive set theoretic lemmas, which seem to be interesting in their own right.

Lemma 2.6. Suppose that $H_{1}$ and $H_{2}$ are closed subgroups of a Polish group $H$. Let $H_{3}=H_{1} \cap H_{2}$. Then, $H_{1} H_{2}$ is a Borel subset of $H$. Moreover, for every $A_{1}=A_{1} H_{3}$ a Borel subset of $H_{1}$ and $A_{2}=H_{3} A_{2}$ a Borel subset of $H_{2}$, we have that $A_{1} A_{2}$ is a Borel subset of $H_{1} H_{2}$.

Proof. Define $f: H_{1} \times H_{3} \times H_{2} \rightarrow H$ by $f\left(h_{1}, h_{3}, h_{2}\right)=h_{1} h_{3} h_{2}$. By [7, Theorem 12.17], we can choose a Borel set $S_{1}$ meeting every coset from $H_{1} / H_{3}$ in exactly one point and a Borel set $S_{2}$ meeting every coset from $H_{3} \backslash H_{2}$ in exactly one point.

Let $f_{0}$ be the function $f$ restricted to $S_{1} \times H_{3} \times S_{2}$. Notice that $f_{0}$ is an injection. Indeed, if $s_{1}, s_{1}^{\prime} \in S_{1}, s_{2}, s_{2}^{\prime} \in S_{2}, h, h^{\prime} \in H_{3}$ and $s_{1} h s_{2}=s_{1}^{\prime} h^{\prime} s_{2}^{\prime}$, then $s_{1}^{\prime-1} s_{1}=h^{\prime} s_{2}^{\prime} s_{2}^{-1} h^{-1} \in H_{1} \cap H_{2}=H_{3}$. Hence, $s_{1}=s_{1}^{\prime}$. Similarly $s_{2}=s_{2}^{\prime}$. So, $h=h^{\prime}$.

Since $S_{1} \times H_{3} \times S_{2}$ is a Borel subset of the Polish space $H_{1} \times H_{3} \times H_{2}$, we get that $f_{0}\left[S_{1} \times H_{3} \times S_{2}\right]=S_{1} H_{3} S_{2}=H_{1} H_{2}$ is a Borel subset of $H$.

To prove the second part of the lemma, first notice that $A_{1}^{\prime}:=A_{1} \cap S_{1}$ and $A_{2}^{\prime}:=A_{2} \cap S_{2}$ are Borel subsets of $H_{1}$ and $H_{2}$, respectively. Hence, $f_{0}\left[A_{1}^{\prime} \times H_{3} \times A_{2}^{\prime}\right]$ is a Borel subset of $H$. On the other hand, $f_{0}\left[A_{1}^{\prime} \times H_{3} \times A_{2}^{\prime}\right]=A_{1}^{\prime} H_{3} A_{2}^{\prime}=A_{1} H_{3} A_{2}=$ $A_{1} A_{2}$. Hence, $A_{1} A_{2}$ is a Borel subset of $H$, which implies that it is a Borel subset of $H_{1} H_{2}$.

Lemma 2.7. Suppose that $H_{1}$ and $H_{2}$ are closed subgroups of a Polish group $H$ such that $H_{1} H_{2}$ is non-meager in its relative topology. Let $H_{3}=H_{1} \cap H_{2}$. Then, for every $A_{1}=A_{1} H_{3} \subseteq_{o} H_{1}$ and $A_{2}=H_{3} A_{2} \subseteq_{o} H_{2}$, we have $A_{1} A_{2} \subseteq_{o} H_{1} H_{2}$. 
Proof. Let us define a function $\psi: H_{1} H_{2} \rightarrow H_{1} / H_{3} \times H_{3} \backslash H_{2}$ by

$$
\psi\left(h_{1} h_{2}\right)=\left(h_{1} H_{3}, H_{3} h_{2}\right),
$$

where $h_{1} \in H_{1}$ and $h_{2} \in H_{2}$.

We check that $\psi$ is well-defined. Suppose $h_{1} h_{2}=h_{1}^{\prime} h_{2}^{\prime}$, where $h_{1}, h_{1}^{\prime} \in H_{1}$ and $h_{2}, h_{2}^{\prime} \in H_{2}$. Then, $h_{1}^{\prime-1} h_{1}=h_{2}^{\prime} h_{2}^{-1} \in H_{1} \cap H_{2}=H_{3}$. Hence, $h_{1} H_{3}=h_{1}^{\prime} H_{3}$ and $H_{3} h_{2}^{\prime}=H_{3} h_{2}$.

To finish the proof, it is enough to show that $\psi$ is continuous. The following claim follows from Lemma 2.6.

Claim 1. $\psi$ is Baire measurable.

By Claim 1 and [7, Theorem 8.38], there is a set $C \subseteq H_{1} H_{2}$ which is a countable intersection of dense open subsets of $H_{1} H_{2}$ such that $\psi \uparrow C$ is continuous.

Claim 2. $\psi$ is continuous.

Proof of Claim 2. Suppose for a contradiction that there are $h_{1}^{i} \in H_{1}$ and $h_{2}^{i} \in H_{2}$, $i \in \omega$, such that for some $h_{1} \in H_{1}$ and $h_{2} \in H_{2}$, we have:

(1) $h_{1}^{i} h_{2}^{i} \longrightarrow h_{1} h_{2}$,

(2) $\left(h_{1}^{i} H_{3}, H_{3} h_{2}^{i}\right) \nrightarrow\left(h_{1} H_{3}, H_{3} h_{2}\right)$.

Notice that (1) and (2) hold for $h h_{1}^{i}, h_{2}^{i} g, h h_{1}, h_{2} g$ instead of $h_{1}^{i}, h_{2}^{i}, h_{1}, h_{2}$ for arbitrary $h \in H_{1}$ and $g \in H_{2}$. Hence, to get a contradiction, it is enough to find $h \in H_{1}$ and $g \in H_{2}$ such that $h h_{1} h_{2} g \in C$ and $h h_{1}^{i} h_{2}^{i} g \in C$ for all $i \in \omega$. So, we will be done if we prove the following:

Subclaim. $\left(\forall h_{1} h_{2} \in H_{1} H_{2}\right)\left(\forall^{*}(h, g) \in H_{1} \times H_{2}\right)\left(h h_{1} h_{2} g \in C\right)$.

Proof of the Subclaim. Since $C^{*}:=\left\{h_{1} h_{2} \in H_{1} H_{2}:\left(\forall^{*}(h, g) \in H_{1} \times H_{2}\right)\left(h h_{1} h_{2} g \in\right.\right.$ $C)\}$ is invariant under multiplication by $H_{1}$ on the left and by $H_{2}$ on the right, it is enough to show that

$$
\left(\exists h_{1} h_{2} \in H_{1} H_{2}\right)\left(\forall^{*}(h, g) \in H_{1} \times H_{2}\right)\left(h h_{1} h_{2} g \in C\right) .
$$

Since $C$ is comeager in $H_{1} H_{2}$, we have

$$
\left(\forall(h, g) \in H_{1} \times H_{2}\right)\left(\forall^{*} h_{1} h_{2} \in H_{1} H_{2}\right)\left(h h_{1} h_{2} g \in C\right) .
$$

So, by the Kuratowski-Ulam theorem [7, Theorem 8.41] we get

$$
\left(\forall^{*} h_{1} h_{2} \in H_{1} H_{2}\right)\left(\forall^{*}(h, g) \in H_{1} \times H_{2}\right)\left(h h_{1} h_{2} g \in C\right) .
$$

Since $H_{1} H_{2}$ is non-meager in its relative topology, we get $(*)$, and we are done.

Lemma 2.8. Suppose that $H_{1}$ and $H_{2}$ are closed subgroups of a Polish group $H$ such that $H_{1} H_{2}$ is non-meager in its relative topology. Let $H_{3}=H_{1} \cap H_{2}$. Then, for every analytic sets $A_{1}=A_{1} H_{3} \subseteq_{n m} H_{1}$ and $A_{2}=H_{3} A_{2} \subseteq_{n m} H_{2}$, we have $A_{1} A_{2} \subseteq_{n m} H_{1} H_{2}$.

Proof. Since $A_{1}$ and $A_{2}$ are analytic subsets of Polish spaces $H_{1}$ and $H_{2}$, by [7, Proposition 14.4, Theorem 21.6] we see that $A_{1} / H_{3} \times H_{3} \backslash A_{2}$ has the Baire property as a subset of $H_{1} / H_{3} \times H_{3} \backslash H_{2}$. Using [7, Lemma 8.43] and the argument from the proof of Proposition 2.3, we get that $A_{1} / H_{3} \times H_{3} \backslash A_{2} \subseteq_{n m} H_{1} / H_{3} \times H_{3} \backslash H_{2}$.

Let us keep the notation from the proof of Lemma 2.7 where it is shown that $\psi$ is continuous, which we use. By the above paragraph, it is enough to prove that $\psi^{-1}[B]$ is non-meager in $H_{1} H_{2}$ whenever $B$ is a non-meager subset of $H_{1} / H_{3} \times$ $H_{3} \backslash H_{2}$ with the Baire property. 
Suppose for a contradiction that $\psi^{-1}[B]$ is meager. Since $B$ is non-meager and has the Baire property, there exists a non-empty open set $U$ and nowhere dense closed sets $F_{n}, n \in \omega$, such that $B \supseteq U \backslash \bigcup_{n} F_{n}$. Then,

$$
\psi^{-1}[B] \supseteq \psi^{-1}[U] \backslash \bigcup_{n} \psi^{-1}\left[F_{n}\right] .
$$

Since $\psi^{-1}[B]$ is meager and $\psi^{-1}[U]$ is open, there is $n_{0} \in \omega$ with $\psi^{-1}\left[F_{n_{0}}\right]$ nonmeager. Indeed, otherwise, $\psi^{-1}[U]$ is open and meager in $H_{1} H_{2}$. Thus, countably many two-sided translates of $\psi^{-1}[U]$ of the form $h_{1} \psi^{-1}[U] h_{2}$ with $h_{1} \in H_{1}$ and $h_{2} \in H_{2}$ cover $H_{1} H_{2}$, and so $H_{1} H_{2}$ is meager in itself, a contradiction.

Since $\psi^{-1}\left[F_{n_{0}}\right]$ is closed, it has a non-empty interior, so countably many of its translates of the form $h_{1} \psi^{-1}\left[F_{n_{0}}\right] h_{2}$ with $h_{1} \in H_{1}$ and $h_{2} \in H_{2}$ cover $H_{1} H_{2}$. Then, the same translates of $F_{n_{0}}$ cover $H_{1} / H_{3} \times H_{3} \backslash H_{2}$, contradicting nowhere density of $F_{n_{0}}$.

To prove (2) in Theorem 2.5, we will need the following corollary of Lemmas 2.7 and 2.8 .

Corollary 2.9. Suppose that $H_{1}$ and $H_{2}$ are closed subgroups of a Polish group $H$ such that $\mathrm{H}_{1} \mathrm{H}_{2}$ is non-meager in its relative topology. Then,

(1) for every $A_{1} \subseteq$ o $H_{1}$, we have $A_{1} H_{2} \subseteq \subseteq_{o} H_{1} H_{2}$,

(2) for every analytic set $A_{1} \subseteq_{n m} H_{1}$, we have $A_{1} H_{2} \subseteq_{n m} H_{1} H_{2}$.

Proof. Replacing $A_{1}$ by $A_{1} H_{3}$ where $H_{3}=H_{1} \cap H_{2}$, we can assume that $A_{1}=A_{1} H_{3}$. Now, apply Lemmas 2.7 and 2.8 for $A_{2}:=H_{2}$.

Now, we are returning to the proof of Theorem 2.5.

Proof of (2) in Theorem 2.5. First consider the case $*=o$. We need to prove that

$$
G_{C} G_{B a} \subseteq_{o} G_{B} \wedge G_{B} G_{A a} \subseteq_{o} G_{A} \Longleftrightarrow G_{C} G_{A a} \subseteq_{o} G_{A} .
$$

$(\Rightarrow)$. Assume $G_{C} G_{B a} \subseteq_{o} G_{B} \wedge G_{B} G_{A a} \subseteq_{o} G_{A}$. Define $H:=G_{A}, H_{1}:=$ $G_{B}, H_{2}:=G_{A a}, A_{1}:=G_{C} G_{B a}$. Then, $H_{1} H_{2}=G_{B} G_{A a} \subseteq_{o} G_{A}$, so it is non-meager in its relative topology. Moreover, $A_{1} \subseteq \subseteq_{o} H_{1}$. Hence, by Corollary 2.9 (1), we get

$$
G_{C} G_{A a}=G_{C} G_{B a} G_{A a}=A_{1} H_{2} \subseteq_{o} H_{1} H_{2}=G_{B} G_{A a} \subseteq_{o} G_{A} .
$$

So, $G_{C} G_{A a} \subseteq_{o} G_{A}$.

$(\Leftarrow)$. Assume $G_{C} G_{A a} \subseteq_{o} G_{A}$. Then, of course, $G_{B} G_{A a} \subseteq_{o} G_{A}$. On the other hand, taking the intersection with $G_{B}$, we get that $G_{C} G_{B a} \subseteq_{o} G_{B}$.

Now, consider the case $*=n m$. We need to prove that

$$
G_{C} G_{B a} \subseteq_{n m} G_{B} \wedge G_{B} G_{A a} \subseteq_{n m} G_{A} \Longleftrightarrow G_{C} G_{A a} \subseteq_{n m} G_{A} .
$$

$(\Rightarrow)$. The proof is similar to the case $*=o$. We only need to check the last implication, namely $G_{C} G_{A a} \subseteq_{n m} G_{B} G_{A a} \subseteq_{n m} G_{A}$ implies $G_{C} G_{A a} \subseteq_{n m} G_{A}$.

Suppose for a contradiction that there are closed and nowhere dense subsets $D_{i}$, $i \in \omega$, of $G_{A}$ such that $G_{C} G_{A a} \subseteq \bigcup_{i \in \omega} D_{i}$. By the assumption that $G_{C} G_{A a} \subseteq_{n m}$ $G_{B} G_{A a}$, there is $i \in \omega$ such that $D_{i} \cap G_{B} G_{A a}$ has a non-empty interior in $G_{B} G_{A a}$. So, countably many translates (by elements of $G_{B}$ on the left and by elements of $G_{A a}$ on the right) of $D_{i}$ cover $G_{B} G_{A a}$. Since $D_{i}$ is nowhere dense in $G_{A}$, we get that $G_{B} G_{A a}$ is meager in $G_{A}$. This is a contradiction.

$(\Leftarrow)$. Assume $G_{C} G_{A a} \subseteq_{n m} G_{A}$. Then, of course, $G_{B} G_{A a} \subseteq_{n m} G_{A}$. It remains to prove that $G_{C} G_{B a} \subseteq_{n m} G_{B}$. 
By Proposition 2.3. we get $G_{C} / G_{A a} \subseteq_{n m} G_{A} / G_{A a}$. Moreover, by [7, Theorem 12.17] we can find a Borel subset $S$ of $G_{C}$ (and hence of $G_{A}$ ) meeting every left coset of $G_{A a} \cap G_{C}$ in exactly one point. Then, the continuous function $\pi: G_{A} \rightarrow G_{A} / G_{A a}$ given by $\pi(g)=g G_{A a}$ is injective on $S$. So, by [7, Theorem 15.1] $\pi[S]=G_{C} / G_{A a}$ is a Borel subset of $G_{A} / G_{A a}$. We conclude that $G_{C} / G_{A a}=D \triangle U$, where $D$ is meager and analytic (even Borel) and $U \neq \emptyset$ is open in $G_{A} / G_{A a}$.

Define $D^{\prime}=\pi^{-1}[D]$ and $U^{\prime}=\pi^{-1}[U]$. Then, we have that $G_{C} G_{A a}=D^{\prime} \triangle U^{\prime}, D^{\prime}$ is meager and analytic [7, Proposition 14.4] and $U^{\prime} \neq \emptyset$ is open in $G_{A}$. Moreover, $U^{\prime} G_{A a}=U^{\prime}$ and $G_{C} \subseteq G_{B}$. Hence, $U^{\prime} \cap G_{B} \neq \emptyset$.

Suppose for a contradiction that $G_{C} G_{B a}$ is meager in $G_{B}$. Since $G_{C} G_{B a}=$ $\left(G_{C} G_{A a}\right) \cap G_{B}=\left(D^{\prime} \triangle U^{\prime}\right) \cap G_{B}=\left(D^{\prime} \cap G_{B}\right) \triangle\left(U^{\prime} \cap G_{B}\right)$ and $U^{\prime} \cap G_{B} \neq \emptyset$ is open in $G_{B}$, we get that $D^{\prime} \cap G_{B} \subseteq_{n m} G_{B}$. We also know that $D^{\prime} \cap G_{B}$ is analytic. Moreover, $G_{B} G_{A a} \subseteq_{n m} G_{A}$, so $G_{B} G_{A a}$ is non-meager in its relative topology. Hence, by Corollary 2.9. (2), we get $\left(D^{\prime} \cap G_{B}\right) G_{A a} \subseteq_{n m} G_{B} G_{A a} \subseteq_{n m} G_{A}$. So, in the same way as in the proof of $(\Rightarrow)$, we get $\left(D^{\prime} \cap G_{B}\right) G_{A a} \subseteq_{n m} G_{A}$. Hence, $D^{\prime} G_{A a} \subseteq_{n m} G_{A}$. Since $D^{\prime} G_{A a}=D^{\prime}$, we see that $D^{\prime} \subseteq_{n m} G_{A}$, a contradiction.

In order to get the existence of $n m$-independent extensions, we assume smallness.

Theorem 2.10 (Existence of $n m$-independent extensions). Let $(X, G)$ be a small Polish structure. Then, for all finite $a \subseteq X$ and $A \subseteq B \subseteq X$, there is $b \in o(a / A)$ such that $b \perp_{A}^{n m} B$.

Before the proof, let us show the following remark.

Remark 2.11. A Polish structure $(X, G)$ satisfies the existence of $*$-independent extensions, where $*=n m$ or $*=o$, iff for all finite $a \subseteq X$ and $A \subseteq B \subseteq X$ there exists $f \in G_{A}$ such that $G_{B} f G_{A a} \subseteq_{*} G_{A}$.

Proof. The existence of $*$-independent extensions is equivalent to the fact that for every finite $a \subseteq X$ and $A \subseteq B \subseteq X$ there is $b \in o(a / A)$ such that $G_{B} G_{A b} \subseteq_{*} G_{A}$, which in turn is equivalent to the conclusion of the remark.

Proof of Theorem 2.10. Consider $a, A, B$ as in the theorem. Let $\left\{a_{i}: i \in I\right\}$ be a countable set of representatives of all orbits over $B$ contained in $o(a / A)$. Then, $o(a / A)=\bigcup_{i \in I} o\left(a_{i} / B\right)$. Take $f_{i} \in G_{A}, i \in I$, such that $f_{i}(a)=a_{i}$.

Claim. $G_{A}=\bigcup_{i \in I} G_{B} f_{i} G_{A a}$.

Proof of Claim. Consider any $f \in G_{A}$. Then, $f(a) \in o\left(a_{i} / B\right)$ for some $i \in I$. Hence, there is $g \in G_{B}$ such that $g(f(a))=f_{i}(a)$. Then, $f_{i}^{-1} g f \in G_{A a}$, so $f \in g^{-1} f_{i} G_{A a} \subseteq G_{B} f_{i} G_{A a}$. Hence, $G_{A} \subseteq \bigcup_{i \in I} G_{B} f_{i} G_{A a}$. The opposite inclusion is obvious.

By virtue of the Claim and the Baire category theorem, there is $i \in I$ such that $G_{B} f_{i} G_{A a} \subseteq_{n m} G_{A}$. So, the proof is completed by Remark 2.11.

We see that the above application of the Baire category theorem works only for $n m$-independence. In Section 4 we will see that the pseudo-arc with the full groups of homeomorphisms is an example of a small Polish structure without the existence of $o$-independence extensions.

One justification for our definition of $n m$-independence is the fact that it satisfies all the fundamental properties (Theorems 2.5] and 2.10) necessary to develop a counterpart of basic geometric stability theory. Another justification is given by the 
next corollary, which shows that in compact [profinite] structures $\mathrm{nm}$-independence coincides with $m$-independence.

Theorem 2.12. Let $(X, G)$ be a Polish structure such that $G$ acts continuously on a separable metrizable space $X$. Let $a, A, B \subseteq X$ be finite. Assume that o $(a / A)$ is non-meager in its relative topology (e.g. it is Polish). Then, $a \downarrow^{*}{ }_{A} B \Longleftrightarrow$ $o(a / A B) \subseteq_{*} o(a / A)$, where $*=o$ or $*=n m$.

Proof. Let $\pi: G_{A} / G_{A a} \rightarrow o(a / A)$ be defined by $\pi\left(g G_{A a}\right)=g a$. Since $o(a / A)$ is non-meager in its relative topology, by Effros' theorem [2, Theorem 2.2.2], we have that $\pi$ is a homeomorphism. Hence, $o(a / A B) \subseteq_{*} o(a / A) \Longleftrightarrow \pi^{-1}[o(a / A B)] \subseteq_{*}$ $\pi^{-1}[o(a / A)] \Longleftrightarrow G_{A B} / G_{A a} \subseteq_{*} G_{A} / G_{A a}$. We finish by using Proposition 2.3.

Corollary 2.13. In every compact structure, o-independence, $n m$-independence and $m$-independence coincide.

In fact, for $\mathrm{nm}$-independence, Theorem 2.12 can be strengthened in the following way.

Theorem 2.14. Let $(X, G)$ be a Polish structure such that $G$ acts continuously on a Hausdorff space $X$. Let $a, A, B \subseteq X$ be finite. Assume that $o(a / A)$ is non-meager in its relative topology. Then, $a \unlhd_{A}^{\beth_{m}^{m}} B \Longleftrightarrow o(a / A B) \subseteq_{n m} o(a / A)$.

Proof. Assume for simplicity that $A=\emptyset$. Let $\pi:=\pi_{\emptyset}: G \rightarrow o(a)$, i.e. $\pi(g)=g a$. By assumption, $\pi$ is continuous. We need to show

$$
\pi^{-1}[o(a / B)] \subseteq_{n m} G \Longleftrightarrow o(a / B) \subseteq_{n m} o(a) .
$$

Before the proof of $(*)$, we need to recall some facts from general topology. We work in a Hausdorff space $Y$. There is a classical result [1, Theorem 25.3] which says that the class of all sets with the Baire property is a $\sigma$-algebra and is closed under the (Souslin) operation $\mathcal{A}$. On the other hand, an easy modification of the proof of [7, Theorem 25.7] gives us that if $f: P \rightarrow Y$ is continuous and $P$ is Polish, then $f[P] \in \mathcal{A}(C L O(Y))$, where $\mathcal{A}(C L O(Y))$ is the family of all sets obtained by application of the operation $\mathcal{A}$ to any family of closed subsets of $Y$. So, we conclude that $f[P]$ has the Baire property.

Now, we return to the proof of $(*)$.

Claim 1. If $M \subseteq_{m} o(a)$, then $\pi^{-1}[M] \subseteq_{m} G$.

Proof. We will be done when we show that $D \subseteq_{n w d} o(a)$ implies $\pi^{-1}[D] \subseteq_{n w d} G$. We can assume that $D$ is closed. Suppose for a contradiction that $\pi^{-1}[D]$ is not nowhere dense in $G$. Since it is closed, it has a non-empty interior. So, there are $g_{i}, i \in \omega$, such that $\bigcup_{i \in \omega} g_{i} \pi^{-1}[D]=G$. Then, $\bigcup_{i \in \omega} g_{i} D=o(a)$. Since $o(a)$ is non-meager in itself, there is $i \in \omega$ such that $g_{i} D \subseteq_{n m} o(a)$, and so $D \subseteq_{n m} o(a)$, a contradiction.

Claim 2. If $N$ has the Baire property as a subset of $o(a)$ and $N \subseteq n m o(a)$, then $\pi^{-1}[N] \subseteq n m$.

Proof. We have $N=U \triangle M$, where $U$ is a non-empty, open and $M$ a meager subset of $o(a)$. So, $\pi^{-1}[N]=\pi^{-1}[U] \triangle \pi^{-1}[M]$. We also have that $\pi^{-1}[U] \neq \emptyset$ is open, and by Claim $1, \pi^{-1}[M]$ is meager in $G$. 
By Claim 1, we get $(\Rightarrow)$ in $(*)$. To show $(\Leftarrow)$, notice that $o(a / B)=\pi\left[G_{B}\right]$ and $G_{B}$ is Polish, so by the remarks immediately below $(*)$, we have that $o(a / B)$ has the Baire property in $o(a)$. We finish by using Claim 2 .

In fact, in the above theorem, instead of assuming that $X$ is equipped with a Hausdorff topology such that the action is continuous, it is enough to assume that there is a Hausdorff topology on $o(a / A)$ such that the action of $G_{A}$ on $o(a / A)$ is continuous.

In Section 4 we will see that the pseudo-arc considered with the group of all homeomorphisms is an example of a small Polish structure where $o$-independence and $n m$-independence differ. Below we give simpler examples of Polish structures in which these two notions differ, but these structures are not small.

Example 1. (i) Let $X=\left(S^{1}\right)^{\omega}$ and $G=\left(\operatorname{Homeo}\left(S^{1}\right)\right)^{\omega}$. We consider the Polish structure $(X, G)$, where $G$ acts naturally on $X$ on the appropriate coordinates. Then, in $(X, G), o$-independence is different from $n m$-independence. Moreover, $(X, G)$ satisfies neither the existence of $o$-independent nor $n m$-independent extensions. Thus, it is not small.

(ii) Let $X=\left(S^{2}\right)^{\omega}$ and $G=\left(\operatorname{Homeo}\left(S^{2}\right)\right)^{\omega}$. Then, we get the same conclusions as in (i), except that now $(X, G)$ satisfies the existence of $n m$-independent extensions.

Proof. (i) Take any $\bar{x}=\left\langle x_{0}, x_{1}, \ldots\right\rangle \in X$ and $\bar{y}=\left\langle y_{0}, y_{1}, \ldots\right\rangle \in X$ such that $x_{i} \neq y_{i}$ for all $i \in \omega$. Then, $o(\bar{x})=X$ and $o(\bar{x} / \bar{y})=S^{1} \backslash\left\{y_{0}\right\} \times S^{1} \backslash\left\{y_{1}\right\} \times \cdots$. So, $o(\bar{x} / \bar{y})$ is non-meager and not open in $o(\bar{x})$. Since $o(\bar{x})=X$ is Polish, by Theorem 2.12 we get that $\bar{x}\rfloor^{n \frac{m}{y}}$ and $\bar{x} \mathcal{L} \bar{y}$. A similar argument shows that $(X, G)$ does not satisfy the existence of $o$-independent extensions.

In order to prove the lack of $n m$-independent extensions, let us consider any $\bar{x}=\left\langle x_{0}, x_{1}, \ldots\right\rangle \in X, \bar{y}=\left\langle y_{0}, y_{1}, \ldots\right\rangle \in X$ and $\bar{z}=\left\langle z_{0}, z_{1}, \ldots\right\rangle \in X$ such that for every $i \in \omega$, the elements $x_{i}, y_{i}$ and $z_{i}$ are pairwise distinct. Take any $\bar{t}=$ $\left\langle t_{0}, t_{1}, \ldots\right\rangle \in X$. Then, $o(\bar{t} / \overline{x y z})$ is a product of singletons and interiors of closed arcs contained in $S^{1}$, so it is meager in $o(\bar{t})=X$. By Theorem 2.12, we get $\bar{t} \mathcal{X}^{\frac{n m}{x} y z}$. (ii) is left to the reader.

\section{BASIC MODEL THEORY}

In this section, we introduce counterparts of some basic notions from stability theory and we investigate their properties. We also study them in a more topological setting of $G$-spaces.

In compact [profinite] structures, definable sets were defined as the sets which are closed and invariant over finite subsets. Since for a Polish structure $(X, G)$ we do not have any topology on $X$, we need another definition of definable sets. Moreover, as in model theory we would like to have (imaginary) names for definable sets for which forking calculus works in the same way as for the real elements. Below we propose definitions of definable sets and imaginary elements that fulfill these requirements.

From now on, we assume that $(X, G)$ is a Polish structure. For $Y \subseteq X^{n}$, we define $\operatorname{Stab}(Y):=\{g \in G: g[Y]=Y\}$. We say that $Y$ is invariant [over a finite set $A]$ if $\operatorname{Stab}(Y)=G\left[\operatorname{Stab}(Y) \supseteq G_{A}\right.$, respectively]. 
Definition 3.1. The imaginary extension, denoted by $X^{e q}$, is the union of all sets of the form $X^{n} / E$, with $E$ ranging over all invariant equivalence relations such that for all $a \in X^{n}, \operatorname{Stab}\left([a]_{E}\right)<_{c} G$. The sets $X^{n} / E$ will be called the sorts of $X^{e q}$.

Remark 3.2. Let $E$ be an invariant equivalence relation on $X^{n}$ whose classes have closed stabilizers in $G$. Then, $G$ induces a group of permutations of $X^{n} / E$, denoted by $G \uparrow X^{n} / E$, which is Polish, and $\left(X^{n} / E, G \uparrow X^{n} / E\right)$ is a Polish structure.

As in model theory, $\left(X^{e q}\right)^{e q}=X^{e q}$, which means that if $E$ is an invariant equivalence relation on a product of sorts $X^{n_{1}} / E_{1} \times \cdots \times X^{n_{k}} / E_{k}$ whose classes have closed stabilizers in $G$, then the set of $E$-classes can be identified with the sort $X^{n_{1}} \times \cdots \times X^{n_{k}} / E^{\prime}$ where

$$
E^{\prime}\left(x_{1}, \ldots, x_{k} ; y_{1}, \ldots, y_{k}\right) \Longleftrightarrow E\left(x_{1} / E_{1}, \ldots, x_{k} / E_{k} ; y_{1} / E_{1}, \ldots, y_{k} / E_{k}\right) .
$$

Definition 3.3. A subset $D$ of $X$ (or more generally of any sort of $X^{e q}$ ) is said to be definable over a finite subset $A$ of $X^{e q}$ (in short $A$-definable) if $D$ is invariant over $A$ and $\operatorname{Stab}(D)<_{c} G$. We say that $D$ is definable if it is definable over some $A$. We say that $d \in X^{e q}$ is a name for $D$ if for every $f \in G$, we have $f[D]=D \Longleftrightarrow f(d)=d$.

Proposition 3.4. Each set definable in $(X, G)$ [or in $\left.X^{e q}\right]$ has a name in $X^{e q}$.

Proof. The proof is similar to the proof of [13, Proposition 1.10]. Suppose $D$ is $a$-definable for some finite $a \in X^{n}$. We define an equivalence relation $E$ on $X^{n}$ by

$$
E\left(a_{1}, a_{2}\right) \Longleftrightarrow\left[a_{1}=a_{2} \vee\left(a_{1}, a_{2}\right) \in S(a, a)\right],
$$

where $S=\{(f, g) \in G \times G: f[D]=g[D]\}$. It is easy to check that $E$ is invariant, every class of $E$ has a closed stabilizer, and $a / E$ is a name for $D$.

Working in $X^{e q}$, we can define $A c l^{e q}$ in the same way as in $X$. Then, the results of Section 2 (including Theorems 2.5 and 2.10) are true in $X^{e q}$ (the only exception is the fact that the Polish structure $(X, G)$ considered in Example 1(ii) does not satisfy the existence of $n m$-independent extensions in $\left.X^{e q}\right)$. However, in the case of a compact [profinite] structure $(X, G)$, both the family of definable sets and $X^{e q}$ computed according to the definitions given in this paper are larger than those computed according to the definitions from [21, 22, 13. For example, any invariant subset of $X$ is definable in our sense, but it is not necessarily closed. Also, if $Y \subseteq X$ is invariant, but not closed, then the equivalence relation $E$ on $X$ with classes $Y$ and $X \backslash Y$ is invariant and $X / E \subseteq X^{e q}$ in our sense, but not in the sense of [21, 22, 13].

We could give more restrictive definitions of definable sets. For example, we could say that an $A$-invariant set $D \subseteq X^{n}$ is definable if for every $a \in X^{n}$, the set $\{g \in G: g a \in D\}$ is closed. Then, the family of definable sets would be closed under finite unions and intersections, but not necessarily under complements (as in compact structures). In the case of compact structures, this notion is also more general than Newelski's notion of definability, but it agrees with it on every orbit. More precisely, an $A$-invariant set $D$ is definable in our sense iff for every $a \in X^{n}$, $o(a) \cap D$ is closed.

Recall some classical definitions. In the whole paper, we assume that compact spaces are Hausdorff by definition.

Definition 3.5. Let $G$ be a Polish group.

(i) A (topological) $G$-space is a Polish structure $(X, G)$ such that $X$ is a topological space and the action of $G$ on $X$ is continuous. 
(ii) A Polish [compact] $G$-space is a $G$-space $(X, G)$, where $X$ is a Polish [compact] space.

In such topological situations, it is natural to consider the same notion of definability as in profinite structures. To distinguish between this notion and our previous notion of definability, we will follow Wagner's terminology [27].

Definition 3.6. Suppose $(X, G)$ is a topological $G$-space. We say that $D \subseteq X^{n}$ is $A$-closed for a finite $A \subseteq X^{e q}$ if it is closed and invariant over $A$. Let $E$ be an invariant equivalence relation on $X^{n}$ whose classes have closed stabilizers in $G$, and equip $X^{n} / E \subseteq X^{e q}$ with the quotient topology. Then, $A$-closed subsets of $X^{n} / E$ are defined as above. We say that $D$ is $*$-closed if it is $A$-closed for some finite $A$.

It would be nice to know that if $(X, G)$ is topologically nice, then so is $\left(X^{e q}, G\right)$. However, in general, taking quotients does not preserve good topological properties like being Hausdorff or Polish. The following considerations tell us that everything looks nice for compact $G$-spaces, which we will be using in Section 5 .

Proposition 3.7. Let $(X, G)$ be a compact $G$-space and $E$ be a $\emptyset$-closed equivalence relation on $X^{n}$. Then, $X^{n} / E$ is compact, and $\left(X^{n} / E, G / G_{X^{n} / E}\right)$ is a compact $G / G_{X^{n} / E^{-}}$space, where $G_{X^{n} / E}=\left\{g \in G: g[a]_{E}=[a]_{E}\right.$ for every $\left.a \in X^{n}\right\}$.

Proof. We can assume $n=1$. The fact that $X / E$ is compact is a standard result in general topology. Let us give a few details. Only the fact that $X / E$ is Hausdorff requires an explanation. Since $E$ is closed and $X$ is compact, the quotient map from $X$ to $X / E$ is closed. Since every compact space is normal and normality is preserved by closed functions, $X / E$ is Hausdorff.

We also have that $G_{X / E}=\bigcap_{x \in X} G_{[x]_{E}}$, which implies that $G_{X / E}<_{c} G$ as each $[x]_{E}$ is a closed subset of $X$. So, $G / G_{X / E}$ is a Polish group.

It remains to show that the action of $G / G_{X / E}$ on $X / E$ is continuous, i.e. the function $\Phi: G / G_{X / E} \times X / E \rightarrow X / E$ given by $\Phi\left(g G_{X / E},[x]_{E}\right)=[g x]_{E}$ is continuous. It is continuous if we equip $G / G_{X / E}$ with the compact-open topology because $X / E$ is compact, and it is easy to see that $G / G_{X / E}$ acts on $X / E$ by homeomorphisms. So, it is enough to show that the quotient topology on $G / G_{X / E}$ is stronger than or equal to the compact-open topology. Take any set $\mathcal{U}$ of the compact-open subbasis on $G / G_{X / E}$, i.e. $\mathcal{U}=\left\{\bar{g} \in G / G_{X / E}: \bar{g}[K] \subseteq U\right\}$, where $K$ is a compact and $U$ is an open subset of $X / E$. Let $\pi_{G}: G \rightarrow G / G_{X / E}$ and $\pi_{X}: X \rightarrow X / E$ be the quotient maps. Then, $K_{1}:=\pi_{X}^{-1}[K]$ is a compact and $U_{1}:=\pi_{X}^{-1}[U]$ is an open subset of $X$. We also have that $\pi_{G}^{-1}[\mathcal{U}]=\left\{g \in G: g\left[K_{1}\right] \subseteq U_{1}\right\}$. Thus, $\pi_{G}^{-1}[\mathcal{U}]$ is open in the compact-open topology on $G$, and hence in the original topology on $G$ because $X$ is compact and the action of $G$ on $X$ is continuous. So, $\mathcal{U}$ is open in the quotient topology on $G / G_{X / E}$.

The above proposition leads to the following definition.

Definition 3.8. Let $(X, G)$ be a compact $G$-space. We define $X^{\text {teq }}$ (topological imaginary extension) as the disjoint union of the spaces $X^{n} / E$ with $E$ ranging over all $\emptyset$-closed equivalence relations on $X^{n}$. The spaces $X^{n} / E$ will be called topological sorts of $X^{\text {teq }}$.

By Proposition 3.7 each topological sort $X / E$ together with the group $G / G_{X / E}$ is a compact $G$-space. If $E$ is $A$-closed for some finite set $A$, then replacing $G$ by $G_{A}, X / E$ can also be treated as a topological sort. We say that $D$ is $A$-closed in 
$X^{t e q}$ if it is $A$-closed in a sort of $X^{t e q}$. Quotients that occur in natural situations are often topological sorts, e.g. if we divide a compact $G$-group (Definition 5.1) by a $*$-closed subgroup.

Proposition 3.9. If $(X, G)$ is a small compact $G$-space, then $X$ is second countable and hence metrizable. Thus, $X$ is a Polish, compact space.

Proof. We need to recall some topological terminology. If $Y$ is a topological space, then a family $\mathcal{N}$ of subsets of $Y$ is said to be a net of $Y$ if for every $y \in Y$ and its open neighborhood $U$, there is $M \in \mathcal{N}$ with $y \in M \subseteq U$. The smallest possible cardinality of a net of $Y$ is called the net weight of $Y$ and is denoted by $n w(Y)$. Of course, for every space $Y$, we have $n w(Y) \leq w(Y)$. For us the following easy fact is essential [3, Twierdzenie 3.1.19]: If $Y$ is compact, then $n w(Y)=w(Y)$.

By this fact, in order to prove that $X$ is second countable, it is enough to show that $n w(X) \leq \aleph_{0}$. Fix a countable basis $\mathcal{U}$ of $G$. Let $\left\{a_{i}: i \in I\right\}$ be a countable set of representatives of all orbits, and $\pi^{i}: G \rightarrow o\left(a_{i}\right), i \in I$, be defined by $\pi^{i}(g)=g a_{i}$. It is easy to see that the countable family $\left\{\pi^{i}[U]: i \in I, U \in \mathcal{U}\right\}$ is a net of $X$.

By [3, Twierdzenie 4.2.8], we get that $X$ is metrizable.

Let us formulate one more topological remark.

Remark 3.10. (i) Let $(X, G)$ be a Polish structure and $D$ be an $A$-definable [or only invariant over $A]$ subset of $X^{e q}$. Then, $\left(D, G_{A} / G_{A D}\right)$ is a Polish structure. Moreover, if $(X, G)$ is small, then $\left(D, G_{A} / G_{A D}\right)$ is also small, and for tuples and subsets of $D, n m$-independence computed in $\left(X, G_{A}\right)$ coincides with $n m$-independence computed in $\left(D, G_{A} / G_{A D}\right)$.

(ii) Let $(X, G)$ be a topological $G$-space. If $D$ is an $A$-closed subset of $X^{n}$ [or $X^{\text {teq }}$, if $X$ is compact], then $\left(D, G_{A} / G_{A D}\right)$ is a topological $G_{A} / G_{A D}$-space.

Proof. (i) Only the fact that the two notions of $n m$-independence coincide requires an explanation. Let $\downarrow^{1}$ and $\downarrow^{2}$ be the notions of $n m$-independence computed in $\left(X, G_{A}\right)$ and $\left(D, G_{A} / G_{A D}\right)$, respectively. Let $a$ be a finite tuple and $A, B$ be finite subsets of $D$.

Assume $a \downarrow_{A}^{1} B$. We need to show $a \bigsqcup_{A}^{2} B$. We have $G_{A B} G_{A a} \subseteq_{n m} G_{A}$. Of course, we also know that $G_{A D}$ is a closed, normal subgroup of $G_{A}$. So, arguing as in the proof of Proposition 2.3. we obtain $\left(G_{A B} / G_{A D}\right)\left(G_{A a} / G_{A D}\right)=$ $\left(G_{A B} G_{A a}\right) / G_{A D} \subseteq_{n m} G_{A} / G_{A D}$, which means that $a \downarrow^{2}{ }_{A} B$.

In order to show that $a \downarrow^{2}{ }_{A} B$ implies $a \perp_{A}^{1} B$, we also proceed as in the proof of Proposition 2.3.

(ii) is very easy.

We come back to the general situation when $(X, G)$ is a Polish structure, but from now on, we assume that $(X, G)$ is small. In fact, more generally, one could only assume that $(X, G)$ satisfies the existence of $n m$-independent extensions and, working in $X^{e q}$, one should also assume that the existence of $n m$-independent extensions holds in $X^{e q}$.

As usual, having a notion of independence satisfying the properties listed in Theorems 2.5 and 2.10, one can define a rank, which has some nice properties. 
Definition 3.11. The $\operatorname{rank} \mathcal{N} \mathcal{M}$ is the unique function from the collection of orbits over finite sets to the ordinals together with $\infty$, satisfying

$$
\begin{gathered}
\mathcal{N} \mathcal{M}(a / A) \geq \alpha+1 \text { iff there is a finite set } B \supseteq A \text { such that } \\
\qquad a \mathcal{X}_{A}^{m m} B \text { and } \mathcal{N} M(a / B) \geq \alpha .
\end{gathered}
$$

The results formulated below follow from a standard forking calculation (e.g. see [26. Lemma 5.1.4 and Theorem 5.1.6]).

Proposition 3.12. Let $a, b, A$ be any finite tuples (subsets) of $X$ or $X^{e q}$.

(1) $a\rfloor_{A}^{r m} b$ implies $\mathcal{N} M(a / A b)=\mathcal{N} M(a / A)$. The converse holds whenever $\mathcal{N} M(a / A)<\infty$.

(2) $\mathcal{N} M(a / b A)+\mathcal{N} M(b / A) \leq \mathcal{N} M(a b / A) \leq \mathcal{N} M(a / b A) \oplus \mathcal{N} M(b / A)$.

(3) Suppose that $\mathcal{N} M(a / A b)<\infty$ and $\mathcal{N} M(a / A) \geq \mathcal{N} M(a / A b) \oplus \alpha$. Then, $\mathcal{N} M(b / A) \geq \mathcal{N} M(b / A a)+\alpha$.

(4) Suppose that $\mathcal{N} M(a / A b)<\infty$ and $\mathcal{N} M(a / A) \geq \mathcal{N} M(a / A b)+\omega^{\alpha} n$. Then, $\mathcal{N} M(b / A) \geq \mathcal{N} M(b / A a)+\omega^{\alpha} n$.

(5) If $a\rfloor_{A}^{r m} b$, then $\mathcal{N} M(a b / A)=\mathcal{N} M(a / b A) \oplus \mathcal{N} M(b / A)$.

As in stable or simple theories, the (in-)equalities in (2)-(5) will be called Lascar inequalities.

Using the existence of $n m$-independent extensions in $X^{e q}$ and Proposition $3.12(1)$, an easy induction yields the following remark.

Remark 3.13. Let $a, A \subseteq X$ be finite. Then, the $\mathcal{N} M$-rank of $o(a / A)$ computed in $X$ is the same as the $\mathcal{N} M$-rank of $o(a / A)$ computed in $X^{e q}$.

Definition 3.14. $(X, G)$ is $n m$-stable if every 1-orbit has ordinal $\mathcal{N} M$-rank.

Remark 3.15. $(X, G)$ is $n m$-stable iff there is no infinite sequence $A_{0} \subseteq A_{1} \subseteq \cdots$ of finite subsets of $X$ and $a \in X$ such that $a \chi_{A_{i}}^{n m} A_{i+1}$ for every $i \in \omega$.

By Lascar inequalities and Remark 3.13, we easily get

Remark 3.16. The following conditions are equivalent to $n m$-stability of $(X, G)$ :

(1) Each $n$-orbit, $n \geq 1$, has ordinal $\mathcal{N} M$-rank.

(2) Each 1-orbit in $X^{e q}$ has ordinal $\mathcal{N} M$-rank.

(3) There is no infinite sequence $A_{0} \subseteq A_{1} \subseteq \cdots$ of finite subsets of $X^{e q}$ and $a \in X^{e q}$ such that $a \mathcal{X}_{A_{i}}^{\text {nom }} A_{i+1}$ for every $i \in \omega$.

\section{Proposition 3.17. TFAE:}

(1) $(X, G)$ is nm-stable.

(2) There is no $a \in X$ and finite sets $A_{0} \subseteq A_{1} \subseteq \cdots \subseteq X$ such that $G_{A_{i+1}} G_{A_{i} a}$ is meager in $G_{A_{i}}$ for every $i \in \omega$.

(3) For every $a \in X$ and finite sets $A_{0} \subseteq A_{1} \subseteq \cdots \subseteq X$, there is $n \in \omega$ such that $G_{A_{n+i+1}} G_{A_{n} a} \subseteq_{n m} G_{A_{n+i}} G_{A_{n} a}$ for every $i \in \omega$.

Proof. (1) $\Leftrightarrow(2)$ is obvious by Proposition 2.3 and Remark 3.15 .

$(1) \Rightarrow(3)$. By Proposition 2.3, Remark 3.15 and transitivity, we can find $n \in \omega$ such that $G_{A_{n+i+1}} G_{A_{n+i} a} \subseteq_{n m} G_{A_{n+i}}$ for every $i \in \omega$. So, by Corollary 2.9.(2) and induction, we easily get that $G_{A_{n+i+1}} G_{A_{n} a} \subseteq_{n m} G_{A_{n+i}} G_{A_{n} a}$ for every $i \in \omega$.

(3) $\Rightarrow(1)$. Take any $a \in X$ and finite sets $A_{0} \subseteq A_{1} \subseteq \cdots \subseteq X$. By (3), there is $n \in \omega$ such that $G_{A_{n+i+1}} G_{A_{n} a} \subseteq_{n m} G_{A_{n+i}} G_{A_{n} a}$ for every $i \in \omega$. Hence, we easily get that $G_{A_{n+i}} G_{A_{n} a} \subseteq_{n m} G_{A_{n}}$ for every $i \in \omega$. So, $\left.a\right\rfloor_{A_{n}}^{n m} A_{n+i+1}$, and hence $a \downarrow_{A_{n+i}}^{n m} A_{n+i+1}$, for every $i \in \omega$. We are done by Remark 3.15 . 
One can adopt the proofs of [20, Proposition 1.1, Corollary 1.2] to conclude the following

Proposition 3.18. Assume $(X, G)$ is nm-stable. Let $a \in X^{e q}, A$ be a finite subset of $X$ and $\alpha \in O r d$. Then, TFAE:

(1) $\mathcal{N} M(a / A) \geq \alpha+1$.

(2) There is a finite set $B \subseteq o(a / A)$ such that $a \mathcal{K}_{A}^{n m} B$ and $\mathcal{N} M(a / A B) \geq \alpha$.

Using this together with Remark 3.10, we get

Corollary 3.19. Assume $(X, G)$ is nm-stable. Let $D$ be definable over $A$, and $a, B \subseteq D$ be finite. Then, $\mathcal{N} M(a / A B)$ computed in $(X, G)$ is equal to $\mathcal{N} M(a / B)$ computed in $\left(D, G_{A} / D_{A D}\right)$.

As it was mentioned in the Introduction, we hope that $n m$-independence, $\mathcal{N} M$ rank and counterparts of some other model theoretic notions may be useful in descriptive set theory or topology. It is worth mentioning here that by Theorem 2.14, if orbits are non-meager in their relative topologies, then $\mathcal{N} M$-rank and $n m$ stability can be expressed in terms of $X$ instead of $G$, and $\mathcal{N} M$-rank measures a 'topological complication' of orbits. More precisely, Theorem 2.14 gives us the following remark.

Remark 3.20. Suppose that $X$ is a Hausdorff space and $G$ acts continuously on $X$. Assume that all orbits over finite sets are non-meager in their relative topologies. Then:

(1) $\mathcal{N} M(a / A) \geq \alpha+1$ iff there is a finite set $B \supseteq A$ such that $o(a / B)$ is meager in $o(a / A)$ and $\mathcal{N} M(a / B) \geq \alpha$.

(2) $(X, G)$ is $n m$-stable iff there is no infinite sequence $A_{0} \subseteq A_{1} \subseteq \cdots$ of finite subsets of $X$ and $a \in X$ such that $o\left(a / A_{i+1}\right)$ is meager in $o\left(a / A_{i}\right)$ for every $i \in \omega$.

By Corollary 2.13, we see that in compact structures, $\mathcal{N} M$-rank and $n m$-stability coincide with $\mathcal{M}$-rank and $m$-stability, respectively.

As in model theory and in profinite structures, one can define a natural pregeometry on an orbit of $\mathcal{N} M$-rank 1 . To introduce this definition, we first need to prove several remarks.

Remark 3.21. For any finite $a, A \subseteq X^{e q}$, we have that $\mathcal{N} M(a / A)=0$ iff $a \in$ $\operatorname{Acl}^{e q}(A)$.

Proof. $(\Rightarrow)$. Assume $\mathcal{N} M(a / A)=0$. Then, $a \perp_{A}^{n m} B$ for every finite $B \subseteq X^{e q}$. By Theorem 2.5) (3), we get $a \in A_{c l}^{e q}(A)$.

$(\Leftarrow)$. Assume $a \in \operatorname{Acl}^{e q}(A)$. By Theorem 2.5(3), we get that $\left.a\right\rfloor_{A}^{n m} B$ for every finite $B \subseteq X^{e q}$. Hence, $\mathcal{N} M(a / A)=0$.

Notice that if $(X, G)$ is a $G$-space and $o(a / A) \subseteq X$ is Polish and countable, then $o(a / A)$ is discrete.

For a finite set $A \subseteq X^{e q}$, we define $A_{c l}^{e q}(B)=A c l^{e q}(A \cup B)$.

Remark 3.22. Assume $\mathcal{N} M(a / A)=1$ and $B$ is a finite subset of $X^{e q}$. Then, $a \in \operatorname{Acl}_{A}^{e q}(B)$ iff $a \chi_{A}^{n m} B$.

Proof. $(\Rightarrow)$. Assume $a \mathcal{K}_{A}^{n m} B$. Then, we have $\mathcal{N} M(a / A B)<\mathcal{N} M(a / A)=1$. Hence, $\mathcal{N} M(a / A B)=0$. By Remark 3.21, we get $a \in A c l_{A}^{e q}(B)$. 
$(\Leftarrow)$. Assume $a \in \operatorname{Acl}_{A}^{e q}(B)$. By Remark 3.21, $\mathcal{N} M(a / A B)=0<\mathcal{N} M(a / A)$. Hence, by Proposition $3.12(1), a \chi_{A}^{n m} B$.

Proposition 3.23. Assume $\mathcal{N} M(a / A)=1$. Then, $\left(o(a / A), A_{c l}^{e q}\right)$ is a pregeometry.

Proof. The proof is the same as in model theory. Let us only check the exchange property. Take any $B \subseteq o(a / A)$ and $b \in o(a / A)$. Consider any $a^{\prime} \in A c l_{A}^{e q}(B b) \backslash$

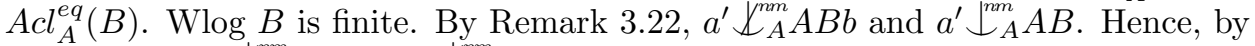
Theorem 2.5. $b \mathbb{L}_{A B}^{n m} a^{\prime}$. So, $b \mathcal{K}_{A}^{n m} B a^{\prime}$, which implies that $b \in A c l_{A}^{e q}\left(B a^{\prime}\right)$.

Definition 3.24. If $D$ is definable over $A$ in $X^{e q}$, put $\mathcal{N} M(D)=\sup \{\mathcal{N} M(d / A)$ : $d \in D\}$.

Let $D$ be definable over $A$. As above, one can show that if $\mathcal{N} M(D)=1$, then $\left(D, A c l_{A}^{e q}\right)$ is a pregeometry.

\section{EXAMPLES}

In this section, we give several examples of small Polish structures. To begin with, notice that all small profinite structures are such examples. This class contains for instance all abelian profinite groups of finite exponent presented as the inverse limit of a system indexed by the natural numbers and considered with the standard structural group of automorphisms [11, Theorem 1.9]. Thus, all such groups with the full group of topological automorphisms are also small Polish structures (see Example 7 in Section (5). Below we give examples which are not profinite.

From a basic topology it follows that whenever $X$ is a compact metric space and $\operatorname{Homeo}(X)$ is the group of all homeomorphisms of $X$ equipped with the compactopen topology, then $(X, \operatorname{Homeo}(X))$ is a Polish structure.

Example 2. (i) For every $n \geq 1$, the Polish structure $\left(S^{n}, \operatorname{Homeo}\left(S^{n}\right)\right)$, where $S^{n}$ is the $n$-dimensional sphere is small of $\mathcal{N} M$-rank 1 .

(ii) For every $n \geq 1$, the Polish structure $\left(\left(S^{1}\right)^{n}, \operatorname{Homeo}\left(\left(S^{1}\right)^{n}\right)\right)$, where $\left(S^{1}\right)^{n}$ is the $n$-dimensional torus is small of $\mathcal{N} M$-rank 1 .

Example 3. The Polish structure $\left(I^{\omega}, \operatorname{Homeo}\left(I^{\omega}\right)\right)$, where $I^{\omega}$ is the Hilbert cube is small of $\mathcal{N} M$-rank 1.

Proof. By [18, Section 6.1, Exercise 2], we know that the action is $n$-transitive for every $n \geq 1$.

Example 4. (i) Let $X=\left(S^{1}\right)^{\omega}$ and $G$ be the group of all homeomorphisms of $X$ respecting the inverse system $S^{1} \longleftarrow S^{1} \times S^{1} \longleftarrow \cdots$ consisting of initial subproducts. Then, $(X, G)$ is a small, non- $n m$-stable Polish structure.

(ii) The Polish structure $\left(\left(S^{1}\right)^{\omega}, H o m e o\left(\left(S^{1}\right)^{\omega}\right)\right.$ is small of $\mathcal{N} M$-rank 1.

Proof. (i) It is easy to see that $G$ is a closed subgroup of Homeo $\left(\left(S^{1}\right)^{\omega}\right)$, and so $(X, G)$ is a Polish structure. The following claim is essential, and we leave it as a non-difficult topological exercise.

Claim. Let $\bar{x}=\left\langle x_{i}\right\rangle_{i \in \omega} \in X$ and $A$ be a finite subset of $X$. If $A \neq \emptyset$, choose an element $\bar{a}=\left\langle a_{i}\right\rangle_{i \in \omega} \in A$ with the longest common beginning with $\bar{x}$. If $\bar{x} \neq \bar{a}$, let $n$ be the smallest natural number such that $x_{n} \neq a_{n}$. Then, we have the following possibilities. 
(1) If $A=\emptyset$, then $o(\bar{x} / A)=X$.

(2) If $A \neq \emptyset$ and $\bar{x} \notin A$, then $o(\bar{x} / A)=\left\{x_{0}\right\} \times \cdots \times\left\{x_{n-1}\right\} \times U \times S^{1} \times S^{1} \times \cdots$ for some open $U \subseteq S^{1}$.

(3) If $\bar{x} \in A$, then $o(\bar{x} / A)=\{\bar{x}\}$.

Smallness is a simple consequence of the Claim once we use the fact that on $S^{1}$ there is no uncountable family of open, pairwise disjoint sets. We also see that 1-orbits are non-meager in their relative topologies. Take any $\bar{x}=\left\langle x_{i}\right\rangle_{i \in \omega} \in X$ and $\overline{x^{j}}=\left\langle x_{i}^{j}\right\rangle_{i \in \omega} \in X, j \in \omega$, such that $x_{j+1}^{j} \neq x_{j+1}$ and $x_{i}^{j}=x_{i}$ for $i \leq j$. By the Claim, we see that for every $n \in \omega$, we have that $o\left(\bar{x} /\left\{\overline{x^{j}}: j \leq n\right\}\right)$ is meager in $o\left(\bar{x} /\left\{\overline{x^{j}}: j \leq n+1\right\}\right)$. Thus, by virtue of Remark 3.20, $(X, G)$ is not $n m$-stable.

(ii) follows from the fact that the action is $n$-transitive for every $n \geq 1$. Smallness also follows immediately from (i). We give a brief sketch of the proof of $n$-transitivity. Take any finite set $A \subseteq X$ and $\bar{x}=\left\langle x_{i}\right\rangle_{i \in \omega} \in X \backslash A$. We need to show that $o(\bar{x} / A)=X \backslash A$. Consider any $\bar{y}=\left\langle y_{i}\right\rangle_{i \in \omega} \in X \backslash A$. There is $n \in \omega$ such that $\left\langle x_{0}, \ldots, x_{n}\right\rangle,\left\langle y_{0}, \ldots, y_{n}\right\rangle \notin A\lceil n+1$. Then, there is a homeomorphism $h$ of $\left(S^{1}\right)^{n+1}$ fixing $A\left\lceil n+1\right.$ and mapping $\left\langle x_{0}, \ldots, x_{n}\right\rangle$ to $\left\langle y_{0}, \ldots, y_{n}\right\rangle$. As in the proof of the Claim, $h$ can be lifted to a homeomorphism of $\left(S^{1}\right)^{\omega}$ fixing $A$ and mapping $\bar{x}$ to $\bar{y}$.

Example 5. The Polish structure $(P, \operatorname{Homeo}(P))$, where $P$ is the pseudo-arc, is small and not $\mathrm{nm}$-stable.

Before the proof, we recall some notions and results about continua. Recall that a continuum is a non-empty, compact, connected metric space. The pseudo$\operatorname{arc} P$ is the unique non-degenerate (i.e. with more than one point), hereditarily indecomposable, chainable continuum (hereditary indecomposablity means that no subcontinuum of $P$ can be written as the union of two proper subcontinua; for the definition of a chainable continuum the reader is referred to [19, Chapter 12]). By hereditary idecomposability, we get that for every $A \subseteq P$, the intersection of all subcontinua of $P$ containing $A$ is the smallest subcontinuum of $P$ containing $A$.

Let $C$ be any non-degenerate continuum (e.g. $P$ ). We say that $C$ is irreducible between subsets $A$ and $B$ if there is no proper subcontinuum of $C$ containing $A$ and $B$. For $p \in C$, we define the composant of $p$, denoted by $\kappa(p)$, as the set of the points $x \in C$ for which there is a proper subcontinuum $A$ of $C$ such that $p, x \in A$.

For every $p \in C, \kappa(p)$ is the union of countably many proper subcontinua of $C$ containing $p$ [19, Proposition 11.14] and $\kappa(p)$ is dense and connected [19, Exercise 5.20]. Moreover, by [19, Exercise 6.19], $C$ is indecomposable iff every proper subcontinuum of $C$ is nowhere dense in $C$. Hence,

$(*)$ If $C$ is indecomposable, then $\kappa(p)$ is meager and dense in $C$ for every $p \in C$.

If $C$ is indecomposable, we define an equivalence relation $E$ on $C$ by

$$
E_{C}(x, y) \Longleftrightarrow y \in \kappa(x) .
$$

By $(*)$ and the Baire category theorem, $E_{C}$ has uncountably many classes (they are just composants). We will need the following two facts, which can be found in [16, Theorem 2, Theorem 6].

Fact 4.1. The pseudo-arc is homeomorphic to each of its non-degenerate subcontinua. 
Fact 4.2. Suppose $H_{1,1}, H_{1,2}, \ldots, H_{1, n}$ are proper subcontinua of the pseudo-arc $P$ and that $P$ is irreducible between each pair of them. Suppose $T$ is a homeomorphism of $H_{1,1} \cup H_{1,2} \cup \cdots \cup H_{1, n}$ onto $H_{2,1} \cup H_{2,2} \cup \cdots \cup H_{2, n}$, where $H_{2,1}, H_{2,2}, \ldots, H_{2, n}$ are proper subcontinua of $P$ such that $P$ is irreducible between each pair of them. Then, $T$ can be extended to a homeomorphism of $P$ onto $P$.

Now, we can prove the following lemma which immediately implies that the Polish structure $(P, \operatorname{Homeo}(P))$ is small.

Lemma 4.3. For every $n \geq 1$, there are only finitely many orbits on $P^{n}$ under the action of Homeo $(P)$.

Proof. The proof is by induction on $n$. The case $n=1$ follows from the fact that the pseudo-arc is homogeneous.

Assume that Lemma 4.3 is true for the tuples of length $<n$ where $n \geq 2$. Suppose for a contradiction that there are infinitely many $n$-tuples $t_{0}=\left(t_{0}^{0}, \ldots, t_{0}^{n-1}\right), t_{1}=$ $\left(t_{1}^{0}, \ldots, t_{1}^{n-1}\right), \ldots$ lying in different orbits under $\operatorname{Homeo}(P)$. Then, there is an infinite subsequence $t_{i_{0}}, t_{i_{1}}, \ldots$ such that for every $j, k \in \omega$, the tuples $t_{i_{j}}$ and $t_{i_{k}}$ are isomorphic with respect to the relation $E_{P}$ via $f_{j k}$ given by $f_{j k}\left(t_{i_{j}}^{l}\right)=t_{i_{k}}^{l}$. Wlog $i_{j}=j$ for every $j \in \omega$. Then, there is $m \leq n-1$ such that for every $i \in \omega,\left\{t_{i}^{0}, \ldots, t_{i}^{n-1}\right\}$ can be partitioned into classes modulo $E_{P}$, say $A_{i}^{0}, \ldots, A_{i}^{m}$, so that $f_{j k}\left[A_{j}^{l}\right]=A_{k}^{l}$ for every $j, k \in \omega$ and $l \leq m$. Let $B_{i}^{0}, \ldots, B_{i}^{m}$ be the smallest proper subcontinua of $P$ containing $A_{i}^{0}, \ldots, A_{i}^{m}$, respectively. Then, $B_{i}^{0}, \ldots, B_{i}^{m}$ are pairwise disjoint proper subcontinua of $P$ such that $P$ is irreducible between each pair of them.

Case $1(m>0)$. By Fact 4.1, for every $i \in \omega$, we can find homeomorphisms $f_{i}^{l}: B_{i}^{l} \rightarrow B_{0}^{l}, l=0, \ldots, m$.

For $l \in\{0, \ldots, m\}$ and $i \in \omega$, let $t_{i, l}$ be the subtuple of $t_{i}$ consisting of all elements of $A_{i}^{l}$. We see that for a fixed $l$, the tuples $f_{i}^{l}\left(t_{i, l}\right), i \in \omega$, of elements of $B_{0}^{l}$ have the same length smaller than $n$. Hence, by Fact 4.1 and the inductive hypothesis, they belong to only finitely many distinct orbits under $\operatorname{Homeo}\left(B_{0}^{l}\right)$. Thus, there are $i \neq j$ such that for every $l \in\{0, \ldots, m\}$, we can find a homeomorphism $g_{l}: B_{0}^{l} \rightarrow B_{0}^{l}$ mapping $f_{i}^{l}\left(t_{i, l}\right)$ to $f_{j}^{l}\left(t_{j, l}\right)$.

Now, we define

$$
T:=\left(f_{j}^{0}\right)^{-1} g_{0} f_{i}^{0} \cup \cdots \cup\left(f_{j}^{m}\right)^{-1} g_{m} f_{i}^{m} .
$$

Then, $T$ is a homeomorphism of $B_{i}^{0} \cup \cdots \cup B_{i}^{m}$ onto $B_{j}^{0} \cup \cdots \cup B_{j}^{m}$ extending $f_{i j}$. Since both $\left\{B_{i}^{0}, \ldots, B_{i}^{m}\right\}$ and $\left\{B_{j}^{0}, \ldots, B_{j}^{m}\right\}$ are collections of proper subcontinua of $P$ such that $P$ is irreducible between each pair of them, by Fact 4.2, $T$ can be extended to an element of $\operatorname{Homeo}(P)$. Since $T\left(t_{i}\right)=f_{i j}\left(t_{i}\right)=t_{j}$, we get a contradiction.

Case $2(m=0)$. Then, $B_{i}^{0}, i \in \omega$, are proper, non-degenerate subcontinua of $P$, so by Fact 4.1 we can find homeomorphisms $f_{i}: B_{i}^{0} \rightarrow B_{0}^{0}$. Let $t_{i}^{\prime}=f_{i}\left(t_{i}\right)$. If we show that there exist $i \neq j$ and a homeomorphism $f: B_{0}^{0} \rightarrow B_{0}^{0}$ with $f\left(t_{i}^{\prime}\right)=t_{j}^{\prime}$, then $T:=f_{j}^{-1} f f_{i}$ is a homeomorphism from $B_{i}^{0}$ onto $B_{j}^{0}$ such that $T\left(t_{i}\right)=t_{j}$, and we finish using Fact 4.2 .

By the minimality of $B_{i}^{0}, i \in \omega$, we see that each tuple $t_{i}^{\prime}, i \in \omega$, meets at least two composants computed within $B_{0}^{0}$. So, applying the argument preceding Case 1 
for $B_{0}^{0}$ and $t_{0}^{\prime}, t_{1}^{\prime}, \ldots$, we come to the situation described in Case 1 , and the proof is completed.

Lemma 4.4. (P, Homeo $(P))$ is not nm-stable.

Proof. Take any $p \in P$. By $(*)$, we can find $p_{0} \in \kappa(p)$ such that $p_{0} \neq p$. Now, choose the smallest proper subcontinuum $P_{0}$ of $P$ containing $p$ and $p_{0}$. Then, $P_{0}$ is also the pseudo-arc and it is nowhere dense in $P$. We see that $P_{0}$ is definable over $\left\{p, p_{0}\right\}$. Let $q_{0}$ be a name of $P_{0}$. By Fact $4.2, o\left(p / q_{0}\right)=P_{0}$, which is nowhere dense in $o(p)=P$. Since $P$ is Polish, Theorem 2.12 implies that $p K_{q_{0}}^{n m}$.

Let us repeat this step within $P_{0}$. By $(*)$, we can find $p_{1} \in \kappa_{P_{0}}(p)$ such that $p_{1} \neq p$, where $\kappa_{P_{0}}$ is $\kappa$ computed within $P_{0}$. Now, choose the smallest proper subcontinuum $P_{1}$ of $P_{0}$ containing $p$ and $p_{1}$. Then, $P_{1}$ is also the pseudo-arc, and it is nowhere dense in $P_{0}$. We see that $P_{1}$ is definable over $\left\{p, p_{0}, p_{1}\right\}$. Let $q_{1}$ be a name of $P_{1}$. By Fact 4.2, $o\left(p / q_{0} q_{1}\right)=P_{1}$, which is nowhere dense in $o\left(p / q_{0}\right)=P_{0}$. Since $P_{0}$ is Polish, Theorem 2.12 implies that $p \mathcal{K}_{q_{0}}^{n m} q_{1}$.

We repeat this procedure and obtain an infinite sequence of imaginaries $q_{0}, q_{1}, \ldots$ such that $p \mathcal{K}_{q_{<i}}^{n m} q_{\leq i}$ for all $i \in \omega$. By Remark 3.16, the proof is completed.

Remark 4.5. For any $p_{0} \in P$, the orbits over $p_{0}$ are $\left\{p_{0}\right\}, \kappa\left(p_{0}\right) \backslash\left\{p_{0}\right\}$ and $P \backslash \kappa\left(p_{0}\right)$.

Proof. By Fact 4.2, we get that if $p \in P \backslash \kappa\left(p_{0}\right)$, then $o\left(p / p_{0}\right)=P \backslash \kappa\left(p_{0}\right)$. Now, assume $p \in \kappa\left(p_{0}\right) \backslash\left\{p_{0}\right\}$. Of course, $o\left(p / p_{0}\right) \subseteq \kappa\left(p_{0}\right) \backslash\left\{p_{0}\right\}$. To show the opposite inclusion, consider any $q \in \kappa\left(p_{0}\right) \backslash\left\{p_{0}\right\}$. Let $B_{p}$ and $B_{q}$ be the smallest subcontinua containing $\left\{p_{0}, p\right\}$ and $\left\{p_{0}, q\right\}$, respectively. By Fact 4.1 and homogeneity of $B_{q}$, there is a homeomorphism $f: B_{p} \rightarrow B_{q}$ fixing $p_{0}$. So, by virtue of Fact 4.2, $f$ can be extended to a homeomorphism from $P$ onto $P$, which will still be denoted by $f$. By the minimality of $B_{p}$ and $B_{q}$, we see that $f(p)$ and $q$ are not in the composant of $p_{0}$ computed within $B_{q}$. So, the first sentence of the proof gives us the existence of a homeomorphism $h: B_{q} \rightarrow B_{q}$ fixing $p_{0}$ and mapping $f(p)$ to $q$. By Fact 4.2 , $h$ can be extended to a homeomorphism from $P$ onto $P$, which will still be denoted by $h$. Then, $h \circ f: P \rightarrow P$ is a homeomorphism fixing $p_{0}$ and mapping $p$ to $q$.

It is not clear how to repeat the proof of Lemma 4.4 without using imaginaries. Keeping the notation from this proof, by Remark 4.5 and $(*)$, we have that $o\left(p / p_{0}\right)=\kappa\left(p_{0}\right) \backslash\left\{p_{0}\right\}$ is meager in $o(p)=P$ and $P$ is Polish, so by Theorem 2.12 , $p X^{n m} p_{0}$. However, by $(*)$ and Effros' theorem (or directly from the comments preceding $(*)), o\left(p / p_{0}\right)=\kappa\left(p_{0}\right) \backslash\left\{p_{0}\right\}$ is meager in its relative topology, so starting from this point we cannot work just with orbits, but we should look at their preimages in $H_{o m e o}(P)$, which is rather complicated.

Remark 4.6. (i) $(P, H o m e o(P))$ does not satisfy the existence of $o$-independent extensions.

(ii) In $(P, H$ omeo $(P))$, the relations $\bigsqcup^{o}$ and $\rfloor^{n m}$ are different.

Proof. (i) Since $P$ is homogeneous, by Theorem 2.12 , it is enough to show that there is $p \in P$ such that every orbit over $p$ is not open in $P$. Take any $p \in P$. Using Remark 4.5, we have that the only orbits over $p$ are $\{p\}, \kappa(p) \backslash\{p\}$ and $P \backslash \kappa(p)$, so we are done by $(*)$.

(ii) It follows from (i), Lemma 4.3 and Theorem 2.10. We can also see it directly. Take any $p \in P$ and $q \notin \kappa(p)$. By Remark 4.5, $o(q / p)=P \backslash \kappa(p)$. So, by $(*)$, we get that $o(q / p)$ is non-meager in $o(q)=P$, and hence, by Theorem $2.12, q \perp^{n m} p$. 
On the other hand, by $(*)$, we see that $P \backslash \kappa(p)$ is not open in $P$, and hence, by Theorem 2.12, $q \ell_{p}$.

We have seen that natural, simple examples of compact (connected) metric spaces with the full group of homeomorphisms are small Polish structures (e.g. $\left.S^{n},\left(S^{1}\right)^{n}, I^{\omega},\left(S^{1}\right)^{\omega}\right)$. Also, a much more complicated continuum, the pseudo-arc, turned out to be small. On the other hand, the main result of [9] says that a certain continuum, called the pseudo-circle, with the full group of homeomorphisms has uncountably many 1-orbits, so it is not small. Thus, one can formulate the following general problem.

Problem 4.7. Classify all small Polish structures of the form $(X, \operatorname{Homeo}(X))$, where $X$ is a compact metric space [continuum].

In the next section, we will give several examples of small Polish groups regarded as Polish structures, i.e. small Polish structures $(H, G)$ such that $H$ is a Polish group and $G$ acts continuously as a group of automorphisms of $H$.

We end up with a remark, which follows from Lascar inequalities and yields examples of small Polish structures of arbitrary finite $\mathcal{N} M$-rank.

Remark 4.8. If $(X, G)$ is a small Polish structure of $\mathcal{N} M$-rank 1 , then for every natural number $n \geq 1,\left(X^{n}, G\right)$ is a small Polish structure of $\mathcal{N} M$-rank $n$.

\section{Small COMPaCt $G$-Groups}

In this paper, all topological groups are Hausdorff by definition. We will be using the following terminology.

Definition 5.1. Let $G$ be a Polish group.

(i) A Polish group structure is a Polish structure $(H, G)$ such that $H$ is a group and $G$ acts as a group of automorphisms of $H$.

(ii) A (topological) $G$-group is a Polish group structure $(H, G)$ such that $H$ is a topological group and the action of $G$ on $H$ is continuous.

(iii) A Polish [compact] $G$-group is a topological $G$-group $(H, G)$, where $H$ is a Polish [compact] group.

Definition 5.2. (i) We say that a group $H$ is definable in a Polish structure $(X, G)$ [or in $\left.X^{e q}\right]$ if $H$ as well as the group operation on $H$ are definable in $(X, G)\left[X^{e q}\right]$.

(ii) We say that a group $H$ is $*$-closed in a $G$-space $(X, G)$ [or in $X^{t e q}$, if $X$ is compact] if $H$ as well as the group operation on $H$ are $*$-closed.

This section is mostly devoted to investigations of small compact $G$-groups. We will give some examples and prove structural results about such groups.

Let us first discuss a notion of a generic orbit in the general context of small Polish group structures or even groups definable in small Polish structures. So, let $H$ be a group definable over $\emptyset$ (i.e. $H$ and the group operation are definable over $\emptyset$ ) in a small Polish structure $(X, G)$ [or in $X^{e q}$. (We can also work with $H$ definable over a finite set $C$, but then we have to replace $G$ by $G_{C}$. Although this depends on the choice of $C$, there is no problem with computations involving $\mathcal{N} M$ ranks of definable sets because the $\mathcal{N} M$-rank of a definable set does not depend on adding parameters.) Then, $\left(H, G / G_{H}\right)$ is a small Polish group structure. For simplicity, one can think about the case when $(X, G)=(H, G)$ is a small Polish group structure.

Let $a \in H$ and $A \subseteq X^{e q}$ be finite. 
Definition 5.3. We say that the orbit $o(a / A)$ is left $n m$-generic (or that $a$ is left $n m$-generic over $A$ ) if for all $b \in H$ with $a\rfloor_{A}^{n m} b$, one has that $b \cdot a \perp^{n m} A, b$. We say that it is right $n m$-generic if, for $b$ as above, we have $a \cdot b \iota^{n m} A, b$. An orbit is $n m$-generic if it is both right and left $n m$-generic.

All the facts about generic [thorn generic] types in simple [rosy] groups which use only formal properties of the underlying independence relation go through in our situation. In particular, Lemma 1.12, Remark 1.20 and Propositions 1.24, $1.26,1.27(1)$ of 4 are true in our context, after replacing $*$ by $n m, U^{*}$ by $\mathcal{N} M$, and assuming that $H / L$ is contained in a sort of $X^{e q}$ (whenever the set $H / L$ of cosets modulo the definable subgroup $L$ occurs) which is always the case when $\left(H, G / G_{H}\right)$ is a $G$-group and $L$ is a $*$-closed subgroup of $H$. For example, we have: if $a$ is left [right] $n m$-generic over $A$, then $a\rfloor^{n m} A$; being left [right] $n m$-generic is preserved under taking restrictions and $n m$-independent extensions; left $\mathrm{nm}$ generic coincides with right $\mathrm{nm}$-generic, and so with $\mathrm{nm}$-generic; in the $\mathrm{nm}$-stable situation, being $n m$-generic means being of maximal $\mathcal{N} M$-rank. However, in order to get the existence of generics [thorn generics] in simple [rosy] groups, one uses suitable stratified local ranks, which are not present in our situation. In fact, even in the rosy context, if we consider an abstract independence relation, it is not clear whether a generic type with respect to this relation exists (see [4, Question 1.19]). In our context, the corresponding question has the following form.

Question 5.4. Let $(G, H)$ be a small Polish group structure. Does $H$ possess an $n m$-generic orbit?

The answer to the above question is positive in a rather large class of structures.

Proposition 5.5. Suppose $(H, G)$ is a small $G$-group, or, more generally, $H$ is a group definable over $C$ in a small Polish structure $(X, G)$ [or in $X^{e q}$ ] such that $\left(H, G_{C} / G_{C H}\right)$ is a $G_{C} / G_{C H}$-group. Assume $H$ is not meager in itself (e.g. $H$ is Polish or compact, or, more generally, Baire). Then, at least one nm-generic orbit in $H$ exists, and an orbit is nm-generic in $H$ iff it is non-meager in $H$.

Proof. Consider the case when $(H, G)$ is a small $G$-group. A similar argument also works in the more general situation described in the proposition.

By smallness and the fact that $H$ is not meager, for any finite $A \subseteq H$, there is an orbit $o=o(h / A)$ which is non-meager in $H$. Let us show that $o$ is $n m$ generic. Consider any $\left.h^{\prime}\right\rfloor_{A}^{n m} h$. We claim that $o\left(h / A, h^{\prime}\right) \subseteq_{n m} H$. Otherwise, there are closed subsets $D_{i}, i \in \omega$, of $H$ with empty interiors such that $o\left(h / A, h^{\prime}\right) \subseteq$ $\bigcup_{i \in \omega} D_{i}$. Then, $\pi_{A}^{-1}\left[o\left(h / A, h^{\prime}\right)\right] \subseteq \bigcup_{i \in \omega} \pi_{A}^{-1}\left[D_{i}\right]$, where $\pi_{A}: G_{A} \rightarrow o(h / A)$ is given by $\pi_{A}(g)=g h$. Since $\pi_{A}^{-1}\left[o\left(h / A, h^{\prime}\right)\right] \subseteq_{n m} G_{A}$ and $\pi_{A}^{-1}\left[D_{i}\right], i \in \omega$, are closed, there is $k$ such that $\pi_{A}^{-1}\left[D_{k}\right]$ has non-empty interior in $G_{A}$. So, countably many left translates of $\pi_{A}^{-1}\left[D_{k}\right]$ by some elements $g_{0}, g_{1}, \cdots \in G_{A}$ cover $G_{A}$. Hence, the sets $g_{i} D_{k}, i \in \omega$, cover $o(h / A)$. Thus, $o(h / A)$ is meager in $H$, a contradiction. From $o\left(h / A, h^{\prime}\right) \subseteq$ nm $H$ we get

$$
o\left(h / A, h^{\prime}\right) h^{\prime} \subseteq_{n m} H \Rightarrow o\left(h h^{\prime} / A, h^{\prime}\right) \subseteq_{n m} H \Rightarrow o\left(h h^{\prime} / A, h^{\prime}\right) \subseteq_{n m} o\left(h h^{\prime}\right) .
$$

By Theorem 2.14, we conclude that $\left.h h^{\prime}\right\rfloor^{n m} A, h^{\prime}$.

We have proved that every orbit which is non-meager in $H$ is $n m$-generic, and so an $n m$-generic orbit exists. Now, suppose that $o(k / A)$ is $n m$-generic. We need to show that $o(k / A) \subseteq_{n m} H$. Take $h \in H$ such that $o(h / A) \subseteq_{n m} H$ and 
$k \longleftarrow_{A}^{n m} h$. Then, $h k \downarrow_{A}^{n m} h$, so $\left.h\right\rfloor_{A}^{n m} k^{-1} h^{-1}$. By the previous paragraph of the proof, we get $o\left(h / A, k^{-1} h^{-1}\right) \subseteq_{n m} H$. So, $o\left(k^{-1} / A, k^{-1} h^{-1}\right) \subseteq_{n m} H$, which implies $o\left(k^{-1} / A\right) \subseteq_{n m} H$, and so $o(k / A) \subseteq_{n m} H$.

The above proposition together with Lascar inequalities for groups 4, Proposition 1.27(1)] easily imply the following corollary, whose direct proof is given below for the reader's convenience. In fact, only point (i) of this corollary will be very useful later. Notice that point (ii) does not require the existence of $n m$-generics.

Corollary 5.6. Let $(H, G)$ be a small $G$-group, or, more generally, $H$ be a group definable over $C$ in a small Polish structure $(X, G)$ [or in $X^{e q}$ ] such that $\left(H, G_{C} / G_{C H}\right)$

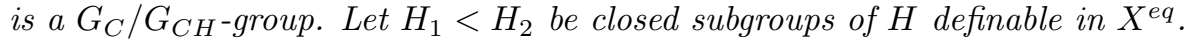

(i) Assume that $H_{1}$ and $H_{2}$ are non-meager in their relative topologies (which holds if $\mathrm{H}$ is Polish or compact). If $\mathrm{H}_{1}<_{n w d} \mathrm{H}_{2}$ and $\mathcal{N} M\left(H_{2}\right)<\infty$, then $\mathcal{N} M\left(H_{1}\right)<\mathcal{N} M\left(H_{2}\right)$.

(ii) If $H_{1}<_{o} H_{2}$, then $\mathcal{N} M\left(H_{1}\right)=\mathcal{N} M\left(H_{2}\right)$.

Proof. For simplicity, let us consider the case when $(H, G)$ is a small $G$-group and $H_{1}$ and $H_{2}$ are $\emptyset$-closed subgroups. A similar argument works in the general situation.

(i) The equivalence relation on $H$ given by $x E y \Longleftrightarrow x H_{1}=y H_{1}$ is $\emptyset$-closed, so $H / H_{1}$ is a sort of $H^{e q}$. Since $H_{1}<_{n w d} H_{2}$ and $H_{2}$ is non-meager in its relative topology, $\left[H_{2}: H_{1}\right]>\aleph_{0}$. By smallness, $\mathrm{Acl}^{e q}(\emptyset) \cap H / H_{1}$ is countable, so there is $h_{2} \in H_{2}$ such that $h_{2} H_{1} \notin A c l^{e q}(\emptyset)$. By Proposition [5.5, we can choose $h_{1} \mathrm{~nm}$-generic in $H_{1}$ over $h_{2}$. Since $h_{2} h_{1} \in h_{2} H_{1}$, we have $h_{2} H_{1} \in d c l\left(h_{2} h_{1}\right)$. So, $h_{2} H_{1} \chi^{n n n} h_{2} h_{1}$. Thus, $\mathcal{N} M\left(H_{2}\right) \geq \mathcal{N} M\left(h_{2} h_{1}\right)>\mathcal{N} M\left(h_{2} h_{1} / h_{2} H_{1}\right) \geq \mathcal{N} M\left(h_{2} h_{1} / h_{2}\right)=\mathcal{N} M\left(h_{1} / h_{2}\right)=$ $\mathcal{N} M\left(H_{1}\right)$.

(ii) First we show that $\left[H_{2}: H_{1}\right] \leq \omega$. Suppose it is not the case. Let $K=H_{2} / H_{1}$ and $G_{0}=G / G_{K}$. Since $H_{1}<_{o} H_{2}$, the quotient topology on $H_{2} / H_{1}$ is discrete. It is easy to check that $\left(K, G_{0}\right)$ is a small $G_{0}$-space. Since $K$ is uncountable, there is an uncountable orbit $o(a)$ in $K$. As the surjection $\pi: G_{0} \rightarrow o(a)$ given by $\pi(g)=g a$ is continuous, the preimages of singletons form a partition of the Polish group $G_{0}$ into uncountably many open sets, a contradiction.

We need to show $\mathcal{N} M\left(H_{2}\right) \leq \mathcal{N} M\left(H_{1}\right)$ (the opposite inequality is obvious). It is enough to prove that for any $h_{2} \in H_{2}$, there is $h_{1} \in H_{1}$ with $\mathcal{N} M\left(h_{2}\right) \leq$ $\mathcal{N} M\left(h_{1}\right)$. Take any $h_{2} \in H_{2}$. Since $H_{2} / H_{1}$ is countable, it is contained in $A c l^{e q}(\emptyset)$. So, $h_{2} \downarrow^{n m} h_{2} H_{1}$. Choose $a \in o\left(h_{2} / h_{2} H_{1}\right)$ so that $a \Psi_{h_{2} H_{1}}^{n m} h_{2}$. Then, $h_{2} \perp^{n m} a$ and $a^{-1} h_{2} \in H_{1}$. Thus, $\mathcal{N} M\left(h_{2}\right)=\mathcal{N} M\left(h_{2} / a\right)=\mathcal{N} M\left(a^{-1} h_{2} / a\right) \leq \mathcal{N} M\left(a^{-1} h_{2}\right)$, and so $h_{1}:=a^{-1} h_{2}$ does the job.

Proposition 5.7. (i) Let $(H, G)$ be a small Polish G-group. Then, any finitely generated subgroup of $H$ is countable and does not have limit points in $H$.

(ii) Let $(H, G)$ be a small compact $G$-group. Then, $H$ is locally finite.

Proof. Let $S \subseteq H$ be finite.

(i) Then, $\overline{\langle S\rangle} \subseteq \operatorname{dcl}(S)$. So, by smallness, $\overline{\langle S\rangle}<H$ is countable. If $\langle S\rangle$ had a limit point, $\overline{\langle S\rangle}$ would be perfect and hence uncountable, a contradiction.

(ii) By compactness, if $\langle S\rangle$ was infinite, then it would have a limit point, and we would get a contradiction as in (i).

The next example is an example of a small Polish (but non-compact) $G$-group. It shows that point (i) of the above proposition cannot be strengthened to get local 
finiteness of $H$. This is one of the reasons why our proofs of structural results for small compact $G$-groups later in this section do not go through for Polish $G$-groups.

Example 6. Let us consider the discrete topology on $\mathbb{Q}$ and the product topology on $\mathbb{Q}^{\omega}$. We consider the additive group structure on $\mathbb{Q}$. Let $A u t^{0}\left(\mathbb{Q}^{\omega}\right)\left[A u t^{0}\left(\mathbb{Q}^{n}\right)\right.$, respectively] be the group of all automorphisms of $\mathbb{Q}^{\omega}\left[\mathbb{Q}^{n}\right.$, respectively] respecting the inverse system $\mathbb{Q} \longleftarrow \mathbb{Q} \times \mathbb{Q} \longleftarrow \cdots$. Then, $A u t^{0}\left(\mathbb{Q}^{\omega}\right)$ can be considered as the inverse limit of the system consisting of $A u t^{0}\left(\mathbb{Q}^{n}\right), n \in \omega$, where on $A u t^{0}\left(\mathbb{Q}^{n}\right)$ we have the pointwise convergence topology. Then, $\left(\mathbb{Q}^{\omega}, A u t^{0}\left(\mathbb{Q}^{\omega}\right)\right)$ is a small Polish $A u t^{0}\left(\mathbb{Q}^{\omega}\right)$-group of $\mathcal{N} M$-rank 1 , and $\mathbb{Q}^{\omega}$ is torsion-free, 0-dimensional and not compact.

Proof. We leave it to the reader to check that $\left(\mathbb{Q}^{\omega}, A u t^{0}\left(\mathbb{Q}^{\omega}\right)\right)$ is a Polish $A u t^{0}\left(\mathbb{Q}^{\omega}\right)$ group. We will show that it is small of $\mathcal{N} M$-rank 1.

The following claim is obvious.

Claim. If $f \in A u t^{0}\left(\mathbb{Q}^{n}\right)$ and $\left\{\eta_{1}, \ldots, \eta_{n}\right\}$ is a basis of $\mathbb{Q}^{n}$ over $\mathbb{Q}$, then for all $\epsilon_{0}, \epsilon_{1}, \ldots, \epsilon_{n}, \epsilon_{0}^{\prime}, \epsilon_{1}^{\prime}, \ldots, \epsilon_{n}^{\prime} \in \mathbb{Q}$ such that $\epsilon_{0}, \epsilon_{0}^{\prime} \neq 0$, there is $\bar{f} \in A u t^{0}\left(\mathbb{Q}^{n+1}\right)$ such that $\bar{f}\left(0 \frown \epsilon_{0}\right)=0 \frown \epsilon_{0}^{\prime}$ and $\bar{f}\left(\eta_{i}^{\frown} \epsilon_{i}\right)=f\left(\eta_{i}\right) \frown \epsilon_{i}^{\prime}, i=1, \ldots, n$.

Now, consider any finite set $A \subseteq \mathbb{Q}^{\omega}$ and $a \in \mathbb{Q}^{\omega}$. Then, either $a \in \operatorname{Lin}(A)$ or there is the largest natural number $n$ such that $a\lceil n \in \operatorname{Lin}(A)\lceil n$. So, by the Claim, we get that either $a \in \operatorname{Lin}(A)$ and then $o(a / A)=\{a\}$, or $a \notin \operatorname{Lin}(A)$ and then $o(a / A)=\left\{\eta \in \mathbb{Q}^{\omega}: \eta\lceil n=a \mid n \wedge \eta \uparrow(n+1) \notin \operatorname{Lin}(A)\lceil(n+1)\}\right.$, where $n$ is the largest $n$ such that $a \mid n \in \operatorname{Lin}(A)\lceil n$. So, each orbit over $A$ is either a singleton from $\operatorname{Lin}(A)$ or an open set. Hence, there are countably many orbits over $A$, so $\left(\mathbb{Q}^{\omega}, A u t^{0}\left(\mathbb{Q}^{\omega}\right)\right)$ is small; we also see that $\mathcal{N} M\left(\mathbb{Q}^{\omega}\right)=1$.

Example 7. (i) If $H$ is a compact metric group, then $\operatorname{Aut}(H)$ is Polish and $(H, A u t(H))$ is a compact $A u t(H)$-group where $\operatorname{Aut}(H)$ is the group of all topological automorphisms of $H$ equipped with the compact-open topology.

(ii) If $(H, G)$ is a small compact $G$-group, then $(H, A u t(H))$ is a small compact $\operatorname{Aut}(H)$-group, where $\operatorname{Aut}(H)$ is defined as in (i).

Proof. In order to show (i), it is enough to check that $A u t(H)$ is a closed subgroup of $H o m e o(H)$, which is an easy exercise.

(ii) follows from (i) and Proposition 3.9

From now on, we assume that $(\boldsymbol{H}, \boldsymbol{G})$ is a small compact $\boldsymbol{G}$-group. The following theorem is proved by Hewitt and Ross [5, Theorem 28.20].

Fact 5.8. Every compact, torsion group is profinite.

This fact together with Proposition 5.7 imply

Corollary 5.9. $H$ is profinite.

Notice that in spite of the fact that $H$ is profinite we are still in a much more general situation than small profinite groups in the sense of Newelski. This is because $G$ is a Polish group which is not necessarily compact. In particular, here we consider the case when $H$ is a profinite group being the inverse limit of a countable system and $G$ is the full group of topological automorphisms of $H$ (then $G$ is Polish but often not compact), which seems more natural from the point of view of group theory and topology than considering only automorphisms preserving a distinguished inverse system. 
The next two remarks will be useful later.

Remark 5.10. For every clopen subset $U$ of $H, S t a b(U)$ is a clopen subgroup of $G$, and so $[G: S t a b(U)] \leq \omega$. In particular, this applies in the case when $U$ is an open subgroup of $H$.

Proof. We see that $U$ is compact. Since $G$ is a group of automorphisms of $H$ acting continuously on $H$, the topology on $G$ must be stronger than or equal to the compact-open topology. Thus, since

$$
\operatorname{Stab}(U)=\{f \in G: f[U] \subseteq U\} \cap\left\{f \in G: f^{-1}[U] \subseteq U\right\}
$$

we get that $\operatorname{Stab}(U)$ is an open subgroup of $G$, and so it is also closed. Since $G$ is Polish, we get $[G: \operatorname{Stab}(U)] \leq \omega$.

Remark 5.11. If $G_{0}$ is a closed subgroup of $G$ of countable index, then $\left(H, G_{0}\right)$ is

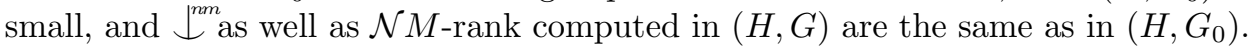

Proof. If $\left(H, G_{0}\right)$ is not small, there are uncountably many $n$-tuples $a_{i}, i \in I$, in different orbits under $G_{0}$. Since $(H, G)$ is small, there are $i_{0} \in I_{0} \subseteq I$ with $\left|I_{0}\right|>\aleph_{0}$ such that for every $i \in I_{0}$, there is $g \in G$ such that $g a_{i_{0}}=a_{i}$. As $\left[G: G_{0}\right] \leq \aleph_{0}$, we can find an uncountable set $I_{1} \subseteq I_{0}$ so that for all distinct $i, j \in I_{1}$, there is $g \in G_{0}$ with $g a_{i}=a_{j}$, a contradiction. So, we have proved that $\left(H, G_{0}\right)$ is small.

Now, suppose that $a \perp_{A}^{n m} B$ in the sense of $\left(H, G_{0}\right)$. Then, $G_{0 A B} G_{0 A a} \subseteq_{n m} G_{0 A}$. Since $G_{0 A}$ is a clopen subgroup of $G_{A}$ and $G_{0 A B} G_{0 A a} \subseteq G_{A B} G_{A a}$, we easily get $G_{A B} G_{A a} \subseteq_{n m} G_{A}$, i.e. $\left.a\right\rfloor_{A}^{n m} B$ in the sense of $(H, G)$.

Finally, assume that $a\rfloor_{A}^{n m} B$ in the sense of $(H, G)$. Then, $G_{A B} G_{A a} \subseteq_{n m} G_{A}$. But $G_{A B} G_{A a}=\bigcup_{i \in I} \bigcup_{j \in J} l_{i} G_{0 A B} G_{0 A a} r_{j}$, where the countable sets $\left\{l_{i}: i \in I\right\}$ and $\left\{r_{j}: j \in J\right\}$ are the sets of representatives of left cosets of $G_{A B}$ modulo $G_{0 A B}$ and right cosets of $G_{A a}$ modulo $G_{0 A a}$, respectively. So, we easily conclude that $G_{0 A B} G_{0 A a} \subseteq_{n m} G_{0 A}$, i.e. $a \complement_{A}^{n m} B$ in the sense of $\left(H, G_{0}\right)$.

We have proved that $\mathbb{L}^{n m}$ is the same in both structures, and so $\mathcal{N} M$-ranks computed in both structures are also the same.

Proposition 5.12. (i) $H$ is the inverse limit of a countable system of finite groups. Thus, it has a countable basis consisting of clopen sets.

(ii) If $G=\operatorname{Aut}(H)$ is the group of all topological automorphisms of $H$, then the topology on $G$ must coincide with the compact-open topology.

(iii) If $G$ is equipped with the compact-open topology, then $G$ has a countable basis of open neighborhoods of id consisting of open subgroups. Thus, $G$ is isomorphic to a closed subgroup of $S_{\infty}$.

Proof. (i) By smallness and Proposition [3.9, $H$ is second countable. As $H$ is also profinite, we get that it has a countable basis of open neighborhoods of $e$ consisting of clopen, normal subgroups $H_{i}, i \in \omega$, with $H_{j}<H_{i}$ whenever $i<j$. So, $H$ is the inverse limit of the countable system consisting of the finite quotients $H / H_{i}, i \in \omega$. Hence, $H$ has a countable basis consisting of clopen sets $U_{i}, i \in \omega$.

(ii) By smallness and Proposition 3.9, $H$ is metrizable (without smallness we would have to assume metrizability). By Example 7, we get that $A u t(H)$ equipped with the compact-open topology is a Polish group. We also have that the given Polish topology on $G=\operatorname{Aut}(H)$ is stronger than or equal to the compact-open topology. So, by [2, Theorem 1.2.6], both topologies on $G$ coincide. 
(iii) By (i), $H$ has a countable basis consisting of clopen sets $U_{i}, i \in \omega$. By Remark 5.10, we have that $\operatorname{Stab}\left(U_{i_{1}} \cup \cdots \cup U_{i_{n}}\right)$ is an open subgroup of $G$ for any $i_{1}, \ldots, i_{n} \in \omega$. So, it is enough to show that every set of the compact-open subbasis on $G$ containing $i d$ contains a subgroup of the form $\operatorname{Stab}\left(U_{i_{1}} \cup \cdots \cup U_{i_{n}}\right)$.

Take any element $\mathcal{U}$ of the compact-open subbasis on $G$ containing $i d$, i.e. $\mathcal{U}=$ $\{f \in G: f[C] \subseteq U\}$, where $C \subseteq H$ is compact, $U \subseteq H$ is open, and $i d \in \mathcal{U}$. Then, $C \subseteq U$. So, by compactness of $C, C \subseteq U_{i_{1}} \cup \cdots \cup \bar{U}_{i_{n}} \subseteq U$ for some $i_{1}, \ldots, i_{n} \in \omega$. Therefore, $\operatorname{Stab}\left(U_{i_{1}} \cup \cdots \cup U_{i_{n}}\right) \subseteq \mathcal{U}$.

Now, the fact that $G$ is isomorphic to a closed subgroup of $S_{\infty}$ follows from [2, Theorem 1.5.1].

Recall the main conjecture concerning small profinite groups.

Conjecture 5.13. Each small profinite group is abelian-by-finite.

In [27], Wagner proved the conjecture by additionally assuming $m$-stability.

Fact 5.14. Each small, $m$-stable profinite group is abelian-by-finite.

In fact, the following three intermediate conjectures are still open.

Conjecture 5.15. Suppose $(H, G)$ is a small profinite group. Then:

(A) $H$ is solvable-by-finite,

(B) if $H$ is solvable-by-finite, then $H$ is nilpotent-by-finite,

(C) if $H$ is nilpotent-by-finite, then $H$ is abelian-by-finite.

Remark 5.16. If $S<H$ is abelian [solvable, nilpotent], then $\bar{S}$ is abelian [solvable, nilpotent].

Below we give examples showing that the generalizations of Conjectures (A), (B) and $(\mathrm{C})$ to the context of small compact $G$-groups are all false. Then, we prove that, for small compact $G$-groups, in the $n m$-stable case Conjecture (A) is true, and in the finite $\mathcal{N} M$-rank case Conjecture (B) is also true. In the rest of this section, by Conjecture 5.15 we will often mean its generalization to the context of small compact $G$-groups.

Example A. Let $S$ be any finite group which is not solvable. Define an action of $S_{\infty}$ on $S^{\omega}$ by

Then,

$$
g\left\langle s_{0}, s_{1}, \ldots\right\rangle=\left\langle s_{g(0)}, s_{g(1)}, \ldots\right\rangle
$$

(i) $S^{\omega}$ is not solvable-by-finite,

(ii) $\left(S^{\omega}, S_{\infty}\right)$ is a small compact $S_{\infty}$-group,

(iii) $\left(S^{\omega}, S_{\infty}\right)$ is not $n m$-stable.

Proof. (i) Let $A$ be a solvable subgroup of $S^{\omega}$. Then, the projections of $A$ on every coordinate are solvable and hence proper subgroups of $S$. So, $\left[S^{\omega}: A\right]$ is infinite.

(ii) The fact that $\left(S^{\omega}, S_{\infty}\right)$ is a compact $S_{\infty}$-group is left to the reader. Now, we will prove smallness. First, notice that for every $\left\langle s_{i}\right\rangle \in S^{\omega}$, we have

$$
\operatorname{Stab}\left(\left\langle s_{i}\right\rangle\right)=\bigcap_{s \in S}\left\{g \in S_{\infty}: g\left\{i \in \omega: s_{i}=s\right\}=\left\{i \in \omega: s_{i}=s\right\}\right\} .
$$

So, for any $\left\langle s_{i}^{1}\right\rangle, \ldots,\left\langle s_{i}^{n}\right\rangle \in S^{\omega}$, we get

$$
\operatorname{Stab}\left(\left(\left\langle s_{i}^{1}\right\rangle, \ldots,\left\langle s_{i}^{n}\right\rangle\right)\right)=\left\{g \in S_{\infty}:(\forall B \in \mathcal{B})(g B=B)\right\},
$$


where $\mathcal{B}$ is the set of all atoms of the finite Boolean algebra generated by the sets $\left\{i \in \omega: s_{i}^{j}=s\right\}, s \in S, j=1, \ldots, n$.

Now, we look at 1-orbits over $\left\{\left\langle s_{i}^{1}\right\rangle, \ldots,\left\langle s_{i}^{n}\right\rangle\right\}$. We get that two elements $\left\langle x_{i}\right\rangle$ and $\left\langle y_{i}\right\rangle$ of $S^{\omega}$ lie in the same orbit over $\left\{\left\langle s_{i}^{1}\right\rangle, \ldots,\left\langle s_{i}^{n}\right\rangle\right\}$ iff for every $s \in S$ and $B \in \mathcal{B}$, the cardinality of $\left\{i \in B: x_{i}=s\right\}$ equals the cardinality of $\left\{i \in B: y_{i}=s\right\}$. Since $S$ and $\mathcal{B}$ are finite and for every $B \in \mathcal{B}$ there are only countably many possibilities for the cardinality of a subset of $B$, we get that there are countably many 1-orbits over $\left\{\left\langle s_{i}^{1}\right\rangle, \ldots,\left\langle s_{i}^{n}\right\rangle\right\}$.

(iii) Let $I_{0} \subseteq I_{1} \subseteq \cdots \subseteq \omega$ be such that $I_{n+1} \backslash I_{n}$ is infinite for every $n \in \omega$. Choose any $s \notin Z(S)$. Define $x_{n}=\left\langle s_{i}^{n}\right\rangle \in S^{\omega}$ by $s_{i}^{n}=s$ if $i \in I_{n}$, and $s_{i}^{n}=e$ if $i \notin I_{n}$. Then, the projection of $C\left(x_{n}\right)$ on the $i$-th coordinate is equal to $C(s) \lesseqgtr S$ if $i \in I_{n}$, and $S$ if $i \notin I_{n}$. Therefore, $C\left(x_{n}\right), n \in \omega$, form a descending sequence of $*$-closed subgroups of $S^{\omega}$ such that $C\left(x_{n+1}\right)<_{n w d} C\left(x_{n}\right)$ for every $n$. So, by Corollary [5.6. $\left(S^{\omega}, S_{\infty}\right)$ is not $n m$-stable.

Example B. Let $S$ be any finite solvable group which is not nilpotent. Define the action of $S_{\infty}$ on $S^{\omega}$ in the same way as in Example A. Then, $\left(S^{\omega}, S_{\infty}\right)$ is a small compact $S_{\infty}$-group which is solvable, not nilpotent-by-finite and not $n m$-stable.

Example C. Let $S$ be any finite nilpotent group which is not abelian. Define the action of $S_{\infty}$ on $S^{\omega}$ in the same way as in Example A. Then, $\left(S^{\omega}, S_{\infty}\right)$ is a small compact $S_{\infty}$-group which is nilpotent, not abelian-by-finite and not $\mathrm{nm}$-stable.

As we will see in the next remark, the above examples also show that some basic properties of small profinite groups are not true for small compact $G$-groups, e.g. invariant subgroups are not necessarily closed.

Remark 5.17. In Examples A, B and C, we have that $|A c l(\emptyset)|=\aleph_{0}$ and $A c l(\emptyset)<S^{\omega}$ is $\emptyset$-invariant but not closed. In fact, $\operatorname{Acl}(\emptyset)$ is dense in $S^{\omega}$.

Proof. It is easy to see that $\operatorname{Acl}(\emptyset)$ consists of those $\left\langle s_{i}\right\rangle \in S^{\omega}$ for which there exists $s \in S$ such that $\left\{i \in \omega: s_{i}=s\right\}$ is cofinite. So, $A c l(\emptyset)$ is dense in $S^{\omega}$ and of cardinality $\aleph_{0}$.

Newelski noticed [21, Remark 4.3] that if $(H, G)$ is a small profinite group where $H=\prod_{n<\omega} H_{n}$ with the $H_{n}$ 's being finite groups is regarded as the inverse limit of all initial subproducts, then almost all $H_{n}$ 's are abelian. Examples A, B and $\mathrm{C}$ show that the same result is not true if $(H, G)$ is a small compact $G$-group. Easy modifications of the proof of [21, Remark 4.3] and the proof of (iii) in Example A yield

Remark 5.18. (i) If $H=\prod_{i \in I} H_{i}$ where the $H_{i}$ 's are finite groups and $(H, G)$ is a small profinite group (but the distinguished inverse system does not necessarily consist of initial subproducts), then almost all $H_{i}$ 's are abelian.

(ii) If $H=\prod_{i \in I} H_{i}$ where the $H_{i}$ 's are finite groups and $(H, G)$ is a small, $n m$-stable, compact $G$-group, then almost all $H_{i}$ 's are abelian.

The above remark shows that Conjecture 5.13 holds for small, $\mathrm{nm}$-stable, compact $G$-groups which are products of finite groups.

Now, we will prove Conjecture 5.15(A) for small, $n m$-stable, compact $G$-groups. Our proof is completely different from the one for small, $m$-stable profinite groups, and it relies on some classical results about profinite groups. In fact, Wagner's proof that each small, $m$-stable profinite group is solvable-by-finite is a part of 
the inductive (on $\mathcal{M}$-rank) proof that each such group is abelian-by-finite 27, Proposition 17], and it uses the inductive hypothesis about abelianity of subgroups of smaller $\mathcal{M}$-rank. There are also some other steps in Wagner's proof of solvability which are not clear in our context, e.g. in our situation invariant subgroups are not necessarily closed.

Theorem 5.19. If $(H, G)$ is a small, nm-stable, compact G-group, then $H$ is solvable-by-finite.

Before the proof, we need to recall some classical facts on profinite groups. There are two main tools that will be used in the proof of Theorem 5.19. Wilson's theorem on the structure of compact torsion groups [28, Theorem 1], and fundamental facts about Frattini subgroups [25, Section 2.8].

Fact 5.20 (Wilson). Let $H$ be a profinite torsion group. Then, $H$ has a finite series

$$
\{e\}=H_{n}<H_{n-1}<\cdots<H_{0}=H
$$

of closed, topologically characteristic subgroups such that each group $H_{i} / H_{i+1}$ is either a pro- $p$ group, for some prime $p$, or a direct product of isomorphic copies of a finite simple group.

Definition 5.21. The Frattini subgroup $\Phi(H)$ of a profinite group $H$ is the intersection of all maximal open subgroups of $H$. Then, $\Phi^{n}(H), n \in \omega$, are defined recursively by $\Phi^{0}(H)=H$ and $\Phi^{k+1}(H)=\Phi\left(\Phi^{k}(H)\right), k \in \omega$.

Fact 5.22. (i) If $H$ is a profinite group, then $\Phi(H)$ is a proper, closed and topologically characteristic subgroup of $H$.

(ii) If $H$ is a pro- $p$ group, then $\Phi(H)=\overline{H^{p}[H, H]}$.

(iii) If $H$ is a pro- $p$ group, then $H$ is finitely generated iff $\Phi(H)<_{o} H$.

Now, we can prove Theorem 5.19

Proof of Theorem 5.19, By Wilson's theorem, there is a normal series

$$
\{e\}=H_{n}<H_{n-1}<\cdots<H_{0}=H
$$

of $\emptyset$-closed subgroups such that each group $H_{i} / H_{i+1}$ is either a pro- $p$ group, for some prime $p$, or a direct product of isomorphic copies of a finite simple group. By Remarks 3.10 and 5.18 we get that each group $H_{i} / H_{i+1}$ is either a pro- $p$ group or a finite group. Replacing $H$ by a clopen subgroup and using Remarks 5.10, 5.11 and 3.10, we can assume that each quotient $H_{i} / H_{i+1}$ is a pro- $p$ group. So, wlog $H$ is a pro- $p$ group.

Of course, we can assume that $H$ is infinite, and then, by Proposition 5.7, we have that $H$ is not finitely generated. So, by Fact $5.22, \Phi(H)$ is a $\emptyset$-closed, nowhere dense subgroup of $H$. We can continue this procedure (taking iterated Frattini subgroups), and by Corollary [5.6, we get that $\Phi^{n}(H)$ is finite for some $n \in \omega$. So, there is $m$ such that $\Phi^{m}(H)=\{e\}$. Since by Fact [5.22, $\Phi^{i}(H) / \Phi^{i+1}(H)$ are abelian, we conclude that $H$ is solvable.

A modification of the proof of Theorem 5.19 yields the following strengthening of this theorem.

Corollary 5.23. If $(H, G)$ is a small, nm-stable, compact $G$-group. Then $H$ has $a *$-closed, solvable subgroup of finite index. 
Proof. We have to modify the first paragraph of the proof of Theorem 5.19. As before, we have a normal series

$$
\{e\}=H_{n}<H_{n-1}<\cdots<H_{0}=H
$$

of $\emptyset$-closed subgroups such that each group $H_{i} / H_{i+1}$ is either a pro- $p$ group or a finite group. We need to show that there is a $*$-closed subgroup $\widetilde{H}$ of finite index in $H$ such that for every $i \leq n-1, \widetilde{H} \cap H_{i} / \widetilde{H} \cap H_{i+1}$ is either a pro- $p$ group or an abelian group. Then we will be done by the second paragraph of the proof of Theorem 5.19]

To obtain $\widetilde{H}$, we will build a sequence $\widetilde{H}_{0}>\cdots>\widetilde{H}_{n-1}$ of $*$-closed, normal, open subgroups of $H$ such that $\widetilde{H}_{j} \cap H_{i} / \widetilde{H}_{j} \cap H_{i+1}$ is either a pro- $p$ group or an abelian group for all $0 \leq i \leq j \leq n-1$. Then, $\widetilde{H}:=\widetilde{H}_{n-1}$ will be as required.

The first step of the construction is obvious: if $H_{0} / H_{1}$ is a pro- $p$ group, we put $\widetilde{H}_{0}:=H_{0}$; otherwise, $H_{0} / H_{1}$ is finite so we can put $\widetilde{H}_{0}:=H_{1}$.

For the induction step, suppose $\widetilde{H}_{0}, \ldots, \widetilde{H}_{j}$ have been constructed for some $j<$ $n-1$. If $K:=\widetilde{H}_{j} \cap H_{j+1} / \widetilde{H}_{j} \cap H_{j+2}$ is a pro- $p$ group, we put $\widetilde{H}_{j+1}=\widetilde{H}_{j}$. Otherwise, let $\widetilde{K}=C_{\widetilde{H}_{j} / \widetilde{H}_{j} \cap H_{j+2}}(K)$. Since $K \triangleleft \widetilde{H}_{j} / \widetilde{H}_{j} \cap H_{j+2}$ is finite, we have that for every $k \in K, C_{\widetilde{H}_{j} / \widetilde{H}_{j} \cap H_{j+2}}(k)$ is a clopen, $*$-closed subgroup of $\widetilde{H}_{j} / \widetilde{H}_{j} \cap H_{j+2}$, and hence $\widetilde{K}$ is a clopen, $*$-closed subgroup of $\widetilde{H}_{j} / \widetilde{H}_{j} \cap H_{j+2}$. Finally, we define $\widetilde{H}_{j+1}$ as the preimage of $\widetilde{K}$ by the natural quotient map.

Next we will prove Conjecture 5.15 (B) for small compact $G$-groups of finite $\mathcal{N} M$ rank. Unlike in the proof of Conjecture 5.15(A), this proof is a modification of the proof of Conjecture 5.15(B) for small, $m$-stable profinite groups [21, Theorem 3.3]. More precisely, the scheme of the proof is the same as in [21, but there are some extra difficulties arising from the fact that in our case: a group generated by an invariant set is not necessarily closed and generated in finitely many steps, we do not know if an increasing sequence of $A$-closed subgroups has to stabilize after finitely many steps, $\operatorname{Acl}(\emptyset)$ is not necessarily finite, etc.

In the following theorem and lemmas, we could skip the assumption that the group is solvable-by-finite because we know it by Theorem [5.19. But we do not do it in order to emphasize that Theorem 5.24 does not rely on Wilson's theorem.

Theorem 5.24. If $(H, G)$ is a small compact $G$-group of finite $\mathcal{N} M$-rank, and $H$ is solvable-by-finite, then $H$ is nilpotent-by-finite.

As in 21, first we need to prove some lemmas. The proof of the next lemma is based on the proof of [21, Lemma 3.4], but it is more complicated in our case.

Lemma 5.25. Suppose $D, H$ are infinite, abelian groups $*$-closed in $X^{\text {teq }}$ for some small compact $G$-space $(X, G)$, and $\mathcal{N} M(H)<\infty$. Assume that $D$ acts on $H$ as a group of automorphisms, and the action is $*$-closed. Then, some open subgroup of $D$ fixes pointwisely an infinite subgroup of $H$.

Proof. Wlog everything is invariant over $\emptyset$. If $D a$ is finite for some $a \in H$, then there is a clopen subgroup $D_{0}$ of $D$ such that $a \in F i x\left(D_{0}\right)$. By Remark 5.10, we easily get that $\operatorname{Stab}_{G}\left(D_{0}\right)$ is a clopen subgroup of countable index in $G$. If Fix $\left(D_{0}\right)$ is infinite, we are done. So, assume $\operatorname{Fix}\left(D_{0}\right)$ is finite. We also have that $\operatorname{Stab}_{G}\left(D_{0}\right) \subseteq \operatorname{Stab}_{G}\left(F i x\left(D_{0}\right)\right)$. So, G $\cdot$ Fix $\left(D_{0}\right)$ is countable, hence Fix $\left(D_{0}\right) \subseteq$ $\operatorname{Acl}(\emptyset)$. Thus, $a \in \operatorname{Acl}(\emptyset)$. 
We have proved that $H_{0}:=\{a \in H: D a$ is finite $\}$ is contained in $A_{c l}^{e q}(\emptyset)$, and so it is countable.

Choose $a \in H$ so that $D a$ is infinite and $H_{1}:=\overline{\langle D a\rangle}<H$ is of minimal possible $\mathcal{N} M$-rank. Then, by virtue of Corollary 5.6, every $D$-invariant, $*$-closed subgroup of $H_{1}$ is either finite or open in $H_{1}$.

Let $R$ be the ring of endomorphisms of $H_{1}$ generated by $D$. Then, $R$ is commutative and every element of $R$ is determined by its value on $a$. Moreover, one can check that the action of $G_{a}$ on $D$ extends to an action by automorphisms on $R$ given by $g\left((-1)^{\varepsilon_{1}} d_{1}+\cdots+(-1)^{\varepsilon_{n}} d_{n}\right)=(-1)^{\varepsilon_{1}} g d_{1}+\cdots+(-1)^{\varepsilon_{n}} g d_{n}$ for $g \in G_{a}, d_{1}, \ldots, d_{n} \in D$ and $\varepsilon_{1}, \ldots, \varepsilon_{n} \in\{0,1\}$, and this extended action preserves the action of $R$ on $H_{1}$. This easily implies that $R$ is locally finite. Indeed, take any $r_{1}, \ldots, r_{n} \in R$, and let $R_{0}=\left\langle r_{1}, \ldots, r_{n}\right\rangle<R$. It is easy to check that $R_{0} a \subseteq \operatorname{dcl}\left(a, r_{1} a, \ldots, r_{n} a\right)$. Hence, $R_{0} a$ must be finite (otherwise, $\overline{R_{0} a}$ would be an uncountable subset of $d c l\left(a, r_{1} a, \ldots, r_{n} a\right)$, a contradiction). Since every element $r_{0} \in R_{0}$ is determined by $r_{0}(a), R_{0}$ is finite.

Now, let $I=\left\{r \in R: r\left[H_{1}\right]\right.$ is finite $\}$. Then, $I$ is a prime ideal of $R$ because for every $r \in R, r\left[H_{1}\right]$ is a $D$-invariant, *-closed subgroup of $H_{1}$, and hence it is either finite or open (so of finite index) in $H_{1}$.

We conclude that $R / I$ is a locally finite integral domain, and hence it is countable. Also, $\widetilde{D}:=D / \operatorname{Stab}_{D}(a)$ can be treated as a subgroup of the multiplicative group of $R$. Therefore, there are $d_{i} \in \widetilde{D}, i \in \omega$, such that

$$
\widetilde{D} \subseteq \bigcup_{i \in \omega} d_{i}+I
$$

Put $D_{i}=\widetilde{D} \cap\left(d_{i}+I\right)$. Then, $\widetilde{D}=\bigcup_{i \in \omega} D_{i}$. If $r \in I$, then $r\left[H_{1}\right]$ is finite and $D$-invariant, and so $r\left[H_{1}\right]<H_{0}$. Hence, for every $i \in \omega$ and $b \in H_{1}$, we have $\left(D_{i}-d_{i}\right) b \subseteq I b \subseteq H_{0}$. Thus, we easily get $D_{i}=\left\{d \in \widetilde{D}:\left(d-d_{i}\right) H_{1} \subseteq H_{0}\right\}$.

For $h \in H_{0}$ and $i \in \omega$, put $K_{h, i}=\left\{(d, b) \in \widetilde{D} \times H_{1}:\left(d-d_{i}\right) b=h\right\}$. We see that $\widetilde{D} \times H_{1}=\bigcup_{h \in H_{0}} \bigcup_{i \in \omega} K_{h, i}$. Since $H_{0}$ is countable and $\widetilde{D} \times H_{1}$ is compact, there are $h \in H_{0}$ and $i \in \omega$ such that $K_{h, i} \subseteq_{n m} \widetilde{D} \times H_{1}$. By continuity of the action of $\widetilde{D}$ on $H_{1}$, we also have that $K_{h, i}$ is closed in $\widetilde{D} \times H_{1}$. Thus, there are $U \subseteq_{o} \widetilde{D}$ and $V \subseteq_{o} H_{1}$ such that $U \times V \subseteq K_{h, i}$.

Let $H_{2}=\left\{b \in H_{1}:\left(U-d_{i}\right) b=\{0\}\right\}<H_{1}$. Then, $H_{2}$ is clopen (and so infinite) in $H_{1}$ because $V-V \subseteq H_{2}$. For every $b \in H_{2}$, we have $U b=d_{i} b$, so $d_{i}^{-1} U b=b$. Hence, $d_{i}^{-1} U \subseteq \widetilde{D}_{H_{2}}:=\left\{d \in \widetilde{D}:\left(\forall b \in H_{2}\right)(d b=b)\right\}<\widetilde{D}$. Since $U \subseteq \subseteq_{o} \widetilde{D}$, we get that $\widetilde{D}_{H_{2}}$ is an open subgroup of $\widetilde{D}$ fixing $H_{2}$.

The proof of the next lemma is similar to the proof of [21, Lemma 3.5]. The only serious difficulty comes from the fact that we do not have a basis of open neighborhoods of $e$ consisting of $\emptyset$-closed subgroups. Our previous remarks take care of that.

Lemma 5.26. Assume that $(H, G)$ is an infinite, small compact $G$-group of finite $\mathcal{N} M$-rank. If $H$ is solvable-by-finite, then some open subgroup of $H$ has an infinite center.

Proof. By Remark [5.16, there is a clopen, solvable subgroup $S$ of $H$. By Remarks 5.10 and 5.11, we can replace $(H, G)$ by $(H, \operatorname{Stab}(S))$. Then, by Remark 3.10 we 
can replace $(H, G)$ by $\left(S, \operatorname{Stab}(S) / \operatorname{Stab}(S)_{S}\right)$, where $\operatorname{Stab}(S)_{S}=\{g \in \operatorname{Stab}(S)$ : $(\forall s \in S)(g(s)=s)\}$. So, wlog $H$ is solvable.

Replacing $H$ by a clopen subgroup (and changing $G$ as above), we can assume that $H$ has an infinite, invariant, normal, abelian subgroup. Taking its closure, we get an infinite, normal, abelian, $*$-closed subgroup of $H$. Let $H_{0}$ be a subgroup of $H$ with all these properties and minimal possible $\mathcal{N} M$-rank, except for the fact that being $*$-closed is with respect to $\left(H, G_{0}\right)$, where $G_{0}$ is a clopen subgroup of $G$ (notice that by Remark 5.11, $\mathcal{N} M$-rank does not depend on the choice of $G_{0}$ ). Wlog $H_{0}$ is $\emptyset$-closed in $(H, G)$.

Let $\left\{L_{i}: i \in I\right\}$ be a basis of clopen, normal subgroups of $H$. Put $H_{i}=H_{0} \cap L_{i}$. It is enough to prove the following

Claim. There exists $i \in I$ such that $\left[H: C_{H}\left(H_{i}\right)\right]<\omega$.

Proof. Suppose the Claim is false. First notice that it is enough to find $i_{0}, i_{1}, \cdots \in I$ such that $C_{H}\left(H_{i_{j}}\right)$ is a subgroup of infinite index in $C_{H}\left(H_{i_{j+1}}\right)$ for each $j \in \omega$. Indeed, since $G$ acts continuously on $H_{0}$, by Remark [5.10, $G_{j}:=\operatorname{Stab}\left(H_{i_{0}}\right) \cap \cdots \cap$ $\operatorname{Stab}\left(H_{i_{j}}\right)$ is a clopen subgroup of $G$. Hence, by Remark 5.11 and Corollary [5.6] in $\left(H, G_{j}\right)$ we have $\mathcal{N} M\left(C_{H}\left(H_{i_{j}}\right)\right)>\mathcal{N} M\left(C_{H}\left(H_{i_{j-1}}\right)\right)>\cdots>\mathcal{N} M\left(C_{H}\left(H_{i_{0}}\right)\right)$, and so $\mathcal{N} M(H) \geq j$. Once again by Remark 5.11, $\mathcal{N} M(H) \geq j$ holds in $(H, G)$ as well. So, $\mathcal{N} M(H) \geq \omega$, a contradiction.

Suppose that $i_{0}, \ldots, i_{n}$ have been chosen. Then, $D:=H / C_{H}\left(H_{i_{n}}\right)$ is an infinite group $\emptyset$-closed in $\left(H, G_{n}\right)^{t e q}$. Of course, $D$ acts on $H_{i_{n}}$ by conjugation. Since $D$ is solvable, there is a clopen, normal subgroup $\widetilde{D}$ of $D$ with an infinite, abelian, invariant under the action of $H$ by conjugation subgroup $D_{1}$, which is $\emptyset$-closed in $\left(H, \operatorname{Stab}_{G_{n}}(\widetilde{D})\right)^{t e q}$. By Lemma 5.25, $D_{i}:=D_{1} \cap\left(L_{i} / C_{H}\left(H_{i_{n}}\right)\right)$ stabilizes an infinite subgroup $H^{*}=F i x\left(D_{i}\right)$ of $H_{i_{n}}$ for some $i \in I$. Since $D_{1}, L_{i}, H_{i_{n}}$ are invariant under the action of $H$ by conjugation, $H^{*}$ is normal in $H$. Moreover, $H^{*}$ is $\emptyset$-closed in $\left(H, S t a b_{G_{n}}(\widetilde{D}) \cap S t a b_{G_{n}}\left(L_{i}\right)\right)$. By Remark 5.10, $\operatorname{Stab}_{G_{n}}(\widetilde{D}) \cap S t a b_{G_{n}}\left(L_{i}\right)$ is a clopen subgroup of $G$, so by the choice of $H_{0}$, we get that $H^{*}$ is open in $H_{0}$. We also see that $\left[C_{H}\left(H^{*}\right): C_{H}\left(H_{i_{n}}\right)\right] \geq\left|D_{i}\right| \geq \omega$. Now, we can choose any $i_{n+1} \in I$ with $H_{i_{n+1}}<H^{*}$ in order to get $\left[C_{H}\left(H_{i_{n+1}}\right): C_{H}\left(H_{i_{n}}\right)\right] \geq \omega$.

Proof of Theorem 5.24. Suppose $H$ is not nilpotent-by-finite. Then, by Lemma 5.26, we can construct infinite sequences $H=H_{0}>H_{1}>\cdots>L_{1}>L_{0}=\{e\}$ such that the $H_{i}$ 's are clopen, normal subgroups of $H$, each $L_{i}$ is a normal subgroup of $H$ which is $*$-closed in $\left(H, \operatorname{Stab}\left(H_{0}\right) \cap \cdots \cap \operatorname{Stab}\left(H_{i}\right)\right)$, and $L_{i+1} / L_{i}=Z\left(H_{i+1} / L_{i}\right)$ is infinite.

Then, the same argument as in the first paragraph of the proof of the Claim in the proof of Lemma 5.26 shows that $\mathcal{N} M(H) \geq \omega$, a contradiction.

By Theorems 5.19 and 5.24, we get the following main result of this section.

Corollary 5.27. If $(H, G)$ is a small compact $G$-group of finite $\mathcal{N} M$-rank, then $H$ is nilpotent-by-finite.

By some standard tricks, Corollary 5.27 implies the following

Corollary 5.28. If $(H, G)$ is a small compact $G$-group of finite $\mathcal{N} M$-rank, then $H$ has a $*$-closed, nilpotent subgroup of finite index.

Proof. By Corollary 5.27 and Remark 5.16, there is a clopen, normal, nilpotent subgroup $\widetilde{H}$ of $H$. The rest of the proof is almost the same as in [24, Theorem 3.17] 
or [14, Remark 3.3(ii)]. Namely, by induction on the nilpotency class, we show that $\widetilde{H}$ has a nilpotent supergroup which is $*$-closed. Notice that in spite of the fact that we do not have icc on centralizers, it is true that for any $S \subseteq H$ such that $[H: C(S)]<\omega, C(S)$ is an intersection of finitely many centralizers of singletons; thus it is $*$-closed, and so is $Z(C(S))$. This is enough to repeat the proof of [14, Remark 3.3(ii)].

Notice that unlike [27, in our proofs we did not need to know that every infinite, small, $n m$-stable compact $G$-group has an infinite, $*$-closed, abelian subgroup. Anyway, the next proposition tells us that this property holds in our case. We prove it modifying slightly the proof of [21, Proposition 3.1].

Proposition 5.29. Every infinite, small, nm-stable compact $G$-group $(H, G)$ has an infinite, *-closed, abelian subgroup.

Proof. Replacing $H$ by an infinite, *-closed subgroup of minimal possible $\mathcal{N} M$ rank, we can assume that $H$ does not have infinite, $*$-closed subgroups of smaller $\mathcal{N} M$-rank. We will show that then $H$ has an abelian, $*$-closed subgroup of finite index.

Since $H$ is locally finite, it has an infinite abelian subgroup [15, Corollary 2.5], say $A$. Taking the closure of $A$, we can assume that $A$ is uncountable. So, there is $a \in A \backslash A \operatorname{Acl}(\emptyset)$ with $C(a)$ infinite and hence open in $H$. Let $d$ be a name for $C(a)$. By Remark [5.10, we see that $d \in A c l^{e q}(\emptyset)$. So, $o(a / d)$ is infinite. Put $V=\overline{\langle o(a / d)\rangle}$. We see that $V$ is an infinite, $*$-closed subgroup of $H$, and hence it is open. Moreover, since $a \in Z(C(a))$, we get $o(a / d) \subseteq Z(C(a))$, so $V<Z(C(a))$. Thus, $V$ is an abelian, *-closed subgroup of finite index in $H$.

Corollary 5.30. Each small, compact G-group of $\mathcal{N} M$-rank 1 is abelian-by-finite.

Let us finish this section with several questions. We have shown that each small, $n m$-stable [of finite $\mathcal{N} M$-rank] compact $G$-group is solvable-by-finite [nilpotent-byfinite, respectively]. It seems that the following should be true.

Conjecture 5.31. Each small, nm-stable compact $G$-group is abelian-by-finite.

The next question is open even for small profinite groups. Notice that Examples $\mathrm{A}, \mathrm{B}$ and $\mathrm{C}$ are not counterexamples to this question.

Question 5.32. Does every small compact $G$-group contain an infinite, abelian, *-closed subgroup?

The structure of small, $n m$-stable Polish $G$-groups is almost completely unknown. Example 6 yields an abelian, Polish $G$-group of $\mathcal{N} M$-rank 1 which is torsion-free and not profinite. Yet, it is still 0-dimensional. It would be interesting to find a non-0-dimensional, small Polish $G$-group. A very interesting question is whether Conjectures 5.15(A), (B) and (C) (with 'by-finite' replaced with 'bycountable') are true for small, $n m$-stable [of finite $\mathcal{N} M$-rank] Polish $G$-groups. In particular, one can ask the following general

Question 5.33. Is it true that every small, $n m$-stable Polish $G$-group is abelianby-countable?

Even the following question is open.

Question 5.34. Is every small, Polish $G$-group of $\mathcal{N} M$-rank 1 abelian-by-countable? 


\section{Final COMments And QUeStions}

In the Introduction, we mentioned the possible directions for further analysis of Polish structures. Below more details are given.

In model theory, there are results, known as group configuration theorems, which say that under some general geometric assumptions one can find a definable group (e.g. [23, Chapters 5, 7]). Such theorems were also proved for small profinite structures [22, Theorem 1.7, Theorem 3.3], or, more generally, for compact structures satisfying the existence of $m$-independent extensions [13. Considerations concerning the existence of a definable (in some sense) group structure are the best example illustrating how stability-theoretic ideas may lead to new aspects in the analysis of classical topological objects. In the context of small Polish structures, it is not clear how to prove such kinds of results. Here we can ask the following general questions.

Question 6.1. Suppose $(X, G)$ is a small Polish structure. When is there a function . : $X \times X \rightarrow X$ definable [or invariant] over a finite subset $A$ of $X$ such that $(X, \cdot)$ is a group?

Question 6.2. Suppose $(X, G)$ is a small Polish structure. When is there an infinite set $Y \subseteq X$ and a function $\cdot: Y \times Y \rightarrow Y$, both definable [or invariant] over a finite subset $A$ of $X$, such that $(Y, \cdot)$ is a group?

The group configuration theorem for small profinite structures [22, Theorem 1.7] yields a partial answer to Question 6.2. Namely, if we additionally assume that $(X, G)$ is profinite and $m$-normal, then in every non-trivial orbit of $\mathcal{M}$-rank 1 there is an open definable group.

So, one of the possible ways for further research is to prove for small Polish structures counterparts of some advanced results from geometric stability theory, e.g. a variant of the group configuration theorem.

The second topic mentioned in the Introduction concerns open questions about the existence of small profinite structures satisfying some additional assumptions. We think it would be interesting to find counterexamples to the generalizations of such questions to the larger class of small Polish structures (e.g. Examples A, B and $\mathrm{C}$ in the previous section are counterexamples to the generalizations of Conjectures $5.15(\mathrm{~A}),(\mathrm{B})$ and $(\mathrm{C})$, respectively). An interesting problem of this kind is

Conjecture 6.3 (NM-gap conjecture). Let $(X, G)$ be a small Polish structure. Then, for every orbit o over a finite subset $A$ of $X$, one has $\mathcal{N} M(o) \in \omega \cup\{\infty\}$.

This conjecture is open in the class of small profinite structures; it was proved only for small, $m$-stable profinite groups [27, Theorem 18]. In the context of small Polish structures, it is open even for small, $\mathrm{nm}$-stable Polish [compact] $G$-groups.

The third topic is Problem 4.7. It seems difficult to give a full classification, but one could at least try to analyze various continua to determine whether they are small and compute their $\mathcal{N} M$-ranks. This could lead to a better understanding of the $\mathcal{N} M$-gap conjecture.

Finally, in Section [5, there are several specific questions about the structure of small Polish [compact] $G$-groups, and it is very likely that progress in this direction can be made. 
At the end, we would like to formulate several descriptive set theoretic (or topological) facts and questions which came up naturally during considerations on Polish structures.

The following fact is Corollary 2.6.8 of [2].

Fact 6.4. The Polish Homeo $\left(I^{\omega}\right)$-space $\left(I^{\omega}, \operatorname{Homeo}\left(I^{\omega}\right)\right)$ is universal for Borel $G$ spaces, i.e. every Borel $G$-space $(X, G)$ can be embedded into $\left(I^{\omega}, H o m e o\left(I^{\omega}\right)\right)$ in the sense that there is a topological isomorphism $\psi: G \rightarrow \psi[G]<_{c} \operatorname{Homeo}\left(I^{\omega}\right)$ and a Borel embedding $\phi: X \rightarrow I^{\omega}$ such that $\phi(g x)=\psi(g) \phi(x)$ for every $g \in G$.

We know that this universal Borel $G$-space is a small Polish structure [Example 3. The following question is a counterpart of the above fact for groups.

Question 6.5. Does there exist a Polish $G$-group $(U, G)$ which is universal for Borel $H$-groups (i.e. every Borel $H$-group $\left(G_{0}, H\right)$ can be embedded into $(U, G)$, preserving the group structure on $\left.G_{0}\right)$ ? If yes, is $(U, G)$ a small Polish $G$-group?

From now on, we fix a Polish group $G$. The following fact comes from 6 , Theorem 0.3 ] and [2, Corollary 2.6.8].

Fact 6.6. There is a Polish $G$-space which is universal for Polish [Borel] $G$-spaces.

Question 6.7. Does there exist a Polish $G$-group which is universal for Polish [Borel] G-groups?

It is obvious that any universal Polish $G$-space (or $G$-group, if it exists) for a fixed $G$ is not small. However, we can ask the following question (or its variants for Polish or Borel $G$-spaces or $G$-groups).

Question 6.8. Does there exist a small Polish structure [group structure] which is universal for small Polish structures [group structures] of the form $(X, G)$ ?

At the very end, we would like to formulate a question concerning small profinite structures. The following is a well-known fact 17.

Fact 6.9. If $G$ is a locally compact group and $(X, G)$ is a normal topological $G$ space, then there is a topological group $H \supseteq X$ such that the topology on $X$ is inherited from $H$, and the action of $G$ on $X$ can be extended to an action on $H$ so that $(H, G)$ is a topological $G$-group.

Question 6.10. Is it true that for every small profinite structure $(X, G)$, one can find a profinite group $H \supseteq X$ such that $X$ is closed in $H$, its topology is inherited from $H$, and the action of $G$ on $X$ can be extended to an action on $H$ so that $(H, G)$ is a small profinite group? if

It is not difficult to construct $H$ so that $(H, G)$ is not necessarily small. Namely,

$$
X=\lim _{\longleftarrow} X_{i}
$$

we can define $H$ as the inverse limit of the system consisting of the linear spaces spanned freely by $X_{i}$ over the two element field $F_{2}$.

Proposition 6.11. If Question 6.10 has the positive answer, then the $\mathcal{M}$-gap conjecture is true for small, m-stable profinite structures. 
Proof. Suppose for a contradiction that $(X, G)$ is a small, $m$-stable profinite structure with an orbit $o$ over some finite set $A$ such that $\mathcal{M}(o) \in \operatorname{Ord} \backslash \omega$. By the assumption, we have that $X$ is $\emptyset$-closed in a small profinite group $(H, G)$.

By [21, Proposition 2.3], the subgroup $\langle X\rangle$ generated by $X$ is $\phi$-closed and generated in finitely many steps. This implies that $(\langle X\rangle, G)$ is a small profinite group. Using the $m$-stability of $(X, G)$, one can easily prove that $(\langle X\rangle, G)$ is also $m$-stable.

Since the $\mathcal{M}$-rank of $o$ computed within $(\langle X\rangle, G)$ is at least $\omega$, we get a contradiction with the fact that $\mathcal{M}$-gap conjecture holds for small, $m$-stable profinite groups [27, Theorem 18].

\section{REFERENCES}

1. A. V. Arhangel'skii (Ed.), General Topology III, Springer, Berlin, Heidelberg, 1995. MR.1416131 (97f:54001)

2. H. Becker, A. S. Kechris, The Descriptive Set Theory of Polish Groups Actions, Cambridge University Press, Cambridge, 1996. MR 1425877 (98d:54068)

3. R. Engelking, Topologia Ogólna, PWN, Warszawa, 1989.

4. C. Ealy, K. Krupiński, A. Pillay, Superrosy groups having finitely satisfiable generics, Ann. Pure Appl. Logic 151 (2008), 1-21. MR2381504 (2009a:03041)

5. E. Hewitt, K. Ross, Abstract Harmonic Analysis II, Springer, Berlin, 1970. MR0262773 $(41: 7378)$

6. G. Hjorth, A universal Polish G-space, Topology Appl. 91 (1999), 141-150. MR.1664504 (2000a:54016)

7. A. S. Kechris, Classical Descriptive Set Theory, Springer, New York, 1995. MR:1321597 (96e:03057)

8. O. Kegel, B. Wehrfritz, Locally Finite Groups, North-Holland, Amsterdam, 1973. MR0470081 $(57: 9848)$

9. J. Kennedy, J. T. Rogers, Jr., Orbits of the pseudocircle, Trans. Amer. Math. Soc. 296 (1986), 327-340. MR837815 (87g:54076)

10. K. Krupiński, Products of finite abelian groups as profinite groups, J. Alg. 288 (2005), 556-582. MR 2146145 (2006g:20042)

11. , Abelian profinite groups, Fund. Math. 185 (2005), 41-59. MR2161751 (2006k:20052)

12. , Profinite structures interpretable in fields, Ann. Pure Appl. Logic 142 (2006), 19-54. MR2250536 (2007k:03090)

13. , Generalizations of small profinite structures, Submitted.

14. __ Fields interpretable in rosy theories, Israel J. Math., to appear.

15. , F. O. Wagner, Small profinite groups and rings, J. Alg. 306 (2006), 494-506. MR.2271348 (2008c:20052)

16. G. L. Lehner, Extending homeomorphisms on the pseudo-arc, Trans. Amer. Math. Soc. 98 (1961), 369-394. MR0120608 (22:11358)

17. M. Megrelishvili, Free topological G-groups, New Zeland J. Math. 25 (1996), 59-72. MR:1398366 (97f:54047)

18. J. van Mill, Infinite-Dimensional Topology. Prerequisites and Introduction, Elsevier Science Publishers, Amsterdam, 1989. MR977744 (90a:57025)

19. S. B. Nadler, Jr., Continuum Theory. An Introduction, Marcel Dekker, New York, 1992. MR.1192552 (93m:54002)

20. L. Newelski, M-gap conjecture and m-normal theories, Israel J. Math. 106 (1998), 285-311. MR:1656901 (2000b:03122)

21. __ Small profinite groups, J. Symb. Logic 66 (2001), 859-872. MR 1833483(2002h:20040)

22. _ Small profinite structures, Trans. Amer. Math. Soc. 354 (2002), 925-943. MR.1867365 (2002j:03038)

23. A. Pillay, Geometric Stability Theory, Oxford University Press, New York, 1996. MR1429864 (98a:03049)

24. B. Poizat, Stable Groups, American Mathematical Society, Providence, 2001. MR:1827833 (2002a:03067) 
25. L. Ribes, P. Zalesskii, Profinite Groups, Springer, Berlin, 2000. MR.1775104 (2001k:20060)

26. F. O. Wagner, Simple Theories, Kluwer Academic Publishers, Dordrecht, 2000. MR.1747713 (2001b:03035)

27. , Small profinite m-stable groups, Fund. Math. 176 (2003), 181-191. MR1971308 (2004d:20029)

28. J. S. Wilson, On the structure of compact torsion groups, Monatsh. Math. 96 (1983), 57-66. MR:721596 (85a:22007)

Instytut Matematyczny Uniwersytetu WrocŁawskiego, Pl. Grunwaldzki 2/4, 50-384 WrocŁaw, Poland - and - Department of Mathematics, University of Illinois at UrbanaChampaign, 1409 W. Green Street, Urbana, Illinois 61801

E-mail address: kkrup@math.uni.wroc.pl 